\title{
Transcriptional profiles of a foliar fungal endophyte (Pestalotiopsis, Ascomycota) and its endohyphal bacterium (Luteibacter, 3 Gammaproteobacteria) in co-culture support sulfur exchange and
}

\section{Justin P. Shaffer ${ }^{1,2+}$, Morgan E. Carter ${ }^{1+}$, Joseph E. Spraker ${ }^{1,3}$, Meara Clark ${ }^{1}$, Brian A.} Smith $^{1}$, Kevin L. Hockett ${ }^{1,4}$, David A. Baltrus ${ }^{1}$, and A. Elizabeth Arnold ${ }^{1,5^{*}}$

${ }^{1}$ School of Plant Sciences, The University of Arizona, Tucson, AZ, USA

${ }^{2}$ Department of Pediatrics, School of Medicine, University of California, La Jolla, CA, USA

${ }^{3}$ Hexagon Bio, Menlo Park, CA, USA

${ }^{4}$ Department of Plant Pathology and Environmental Microbiology, Pennsylvania State University, PA, USA

${ }^{5}$ Department of Ecology and Evolutionary Biology, The University of Arizona, Tucson, AZ, USA

\section{${ }^{+}$Co-first authors.}

* Correspondence:

Dr. A. Elizabeth Arnold

Arnold@ag.arizona.edu

303 Forbes Building

1140 E. South Campus Drive

Tucson, AZ 85721

+1 (520) 344-2327 (telephone)

+1 (520) 621-7186 (fax)

\section{Running title.}

Gene expression in a fungus-bacterium co-culture

\section{Conflict of interest.}

36 The authors declare no conflict of interest. 


\section{ABSTRACT}

38 Symbiosis with bacteria is widespread among eukaryotes, including fungi. Bacteria that live

39 within fungal mycelia (endohyphal bacteria) occur in many plant-associated fungi, including

40 diverse Mucoromycota and Dikarya. Pestalotiopsis sp. 9143 is a filamentous ascomycete

41 isolated originally as a foliar endophyte of Platycladus orientalis (Cupressaceae). It is infected

42 naturally with the endohyphal bacterium Luteibacter sp. 9143, which influences auxin and

43 enzyme production by its fungal host. Previous studies have used transcriptomics to examine

44 similar symbioses between endohyphal bacteria and root-associated fungi such as arbuscular

45 mycorrhizal fungi and plant pathogens. However, currently there are no gene expression studies

46 of endohyphal bacteria of Ascomycota, the most species-rich fungal phylum. We developed

47 methods for assessing gene expression by Pestalotiopsis sp. and Luteibacter sp. when grown in

48 co-culture and when each was grown axenically. Our assays showed that the density of

49 Luteibacter sp. in co-culture was greater than in axenic culture, but the opposite was true for the

50 Pestalotiopsis sp. Dual RNA-seq data demonstrate that growing in co-culture modulates

51 developmental and metabolic processes in both the fungus and bacterium, potentially through

52 changes in the balance of organic sulfur via methionine acquisition. Our analyses also suggest an

53 unexpected, potential role of the bacterial type VI secretion system in symbiosis establishment,

54 expanding current understanding of the scope and dynamics of fungal-bacterial symbioses.

\section{TWEET}

57 When in co-culture, Luteibacter downregulates motility and upregulates a T6SS. Gene

58 expression changes in its host, Pestalotiopsis, suggest the bacterium impacts fungal cell structure 59 and methionine availability. 


\section{IMPORTANCE}

61 Interactions between microbes and their hosts have important outcomes for host- and

62 environmental health. Foliar fungal endophytes that infect healthy plants can harbor facultative

63 endosymbionts called endohyphal bacteria, which can influence the outcome of plant-fungus

64 interactions. These bacterial-fungal interactions can be influential but are poorly understood,

65 particularly from a transcriptome perspective. Here, we report on a comparative, dual RNA-seq

66 study examining the gene expression patterns of a foliar fungal endophyte and a facultative

67 endohyphal bacterium when cultured together vs. separately. Our findings support a role for the

68 fungus in providing organic sulfur to the bacterium, potentially through methionine acquisition,

69 and potential involvement of a bacterial type VI secretion system in symbiosis establishment.

70 This work adds to the growing body of literature characterizing endohyphal bacterial-fungal

71 interactions, with a focus on a model facultative bacterial-fungal symbiosis in two species-rich

72 lineages, the Ascomycota and Proteobacteria.

73

74 KEYWORDS: bacterial-fungal interactions, culture conditions, endobacteria, gene expression,

75 interdomain, interkingdom, Platycladus orientalis, RNAseq, symbiosis, transcriptomics 


\section{INTRODUCTION}

77 Symbioses between eukaryotes and bacteria are widespread, with profound impacts ranging from

78 the benefits of the gut microbiome with respect to human health to the cost of plant pathogens on

79 global agriculture (Lynch and Hsiao, 2019; Savary et al., 2019). The molecular mechanisms

80 underlying relationships ranging from antagonism to mutualism have been studied for decades in

81 animals and plants, including the ways in which pathogenic and beneficial microbes establish in

82 a new host. Although ubiquitous in nature, bacterial-fungal interactions remain relatively poorly

83 understood, despite growing knowledge of their contributions to the emergent properties of

84 microbiomes (Araldi-Brondolo et al., 2017; Steffan et al., 2020). For example, bacteria living

85 with fungi inhabiting plant roots and leaves can influence fungal phenotypes including growth,

86 reproduction, and pathogenicity, as well as the outcomes of plant-fungus interactions (Bianciotto

87 et al. 2004; Partida-Martinez \& Hertweck 2005; Partida-Martinez et al. 2007a; Lumini et al.

88 2007; Shaffer et al. 2018).

Despite "bacterium-like organelles" being discovered in arbuscular mycorrhizal fungi

90 (AMF) decades ago (MacDonald et al., 1982), only recently have endohyphal bacteria (EHB)

91 been identified living intracellularly in diverse plant-associated fungi. To date, members of the

92 Mucoromycota, Basidiomycota, and Ascomycota have been identified as hosts to EHB,

93 including Proteobacteria, Firmicutes, Tenericutes, Bacteroidetes, and others (Bianciotto et al.

94 2003; Partida-Martinez et al. 2007b; Hoffman \& Arnold 2010; Desiro et al. 2015; Shaffer et al.

95 2016). Thus, both the capacity of bacteria to live within fungal hyphae, and the capacity of

96 diverse fungi to harbor bacterial endosymbionts, are phylogenetically widespread and

97 functionally diverse (Araldi-Brondolo et al., 2017; Pawlowska et al., 2018). For example, one

98 EHB associated with AMF, Candidatus Glomeribacter gigasporarum (Betaproteobacteria), is a 
99 vertically transmitted, obligate biotroph with a reduced genome (Bianciotto et al., 2003;

100 Bianciotto et al., 2004). In contrast, diverse EHB cultured from- or observed in ectomycorrhizal

101 fungi and foliar fungal endophytes appear to be facultatively associated with fungal hosts, with

102 relatively unreduced genomes (Izumi et al., 2006; Hoffman and Arnold, 2010; Baltrus et al.,

103 2017). Even among these facultative interactions, the impacts on fungal hosts by EHB include

104 alterations in carbon use, growth of germinating spores, degradation of plant cell wall

105 compounds, and sporulation (Lumini et al., 2007; Partida-Martinez et al., 2007a; Arendt, 2015;

106 Shaffer et al., 2017). The metabolic, proteomic, and transcriptomic changes that facilitate these

107 associations, and associated bacterial and fungal phenotypes, are not well known. Changes to

108 fungal traits by the presence of a bacterial symbiont may impact other organisms, such as a plant

109 host through increased virulence (Obasa et al., 2017) or plant growth promotion (Hoffman et al.,

110 2013; Guo et al., 2017a). Therefore, understanding these bacterial-fungal interactions will

111 expand knowledge of fungal ecology more broadly.

112 Mycetohabitans spp. (formerly Burkholderia, Betaproteobacteria) and their host,

113 Rhizopus microsporus (Mucoromycotina), represent one emerging model system for EHB based

114 on the ability to independently culture and reintroduce the partners in vitro. Metabolic and

115 transcriptomic studies have revealed changes in fungal lipid metabolism underlying their

116 partnership formation, and a lack of reactive oxygen species burst in compatible partners

117 (Lastovetsky et al., 2016; Lastovetsky et al., 2020). The unique requirement of Mycetohabitans

118 spp. for $R$. microsporus sporulation provides a context for probing genes involved in fungal

119 reproduction in a genetically recalcitrant fungal clade (Mondo et al., 2017). Essential bacterial

120 genes for symbiosis establishment have been identified in Mycetohabitans spp., namely a type II

121 and type III secretion system (T2SS, T3SS) required for invading fungal hyphae (Lackner et al., 
122 2011; Moebius et al., 2014). The T2SS secretes chitinases critical for bacterial entry, but only

123 one T3SS effector protein has been characterized, and it is not required for the establishment of

124 symbiosis (Moebius et al., 2014; Carter et al., 2020). Yet, many EHB outside of the

125 Burkholderiales lack one or both the T2SS and T3SS (Araldi-Brondolo et al., 2017; Baltrus et

126 al., 2017). Indeed, the phylogenetic diversity of both EHB and their fungal hosts suggests

127 bacteria can use many yet undiscovered methods to establish in a given fungus, with a variety of

128 potential outcomes. For example, a lipopeptide produced by Ralstonia solanacearum,

129 ralsolamycin, induces chlamydospore formation in- and facilitates invasion of multiple fungi

130 (Spraker et al., 2016).

An additional emerging model for EHB is Luteibacter sp. 9143 (Xanthomonadaceae,

132 Gammaproteobacteria) and its host, the foliar fungal endophyte Pestalotiopsis sp. 9143

133 (Sporocadaceae, Xylariales, Ascomycota) (Hoffman and Arnold, 2010). The Luteibacter-

134 Pestalotiopsis interaction represents the typical facultative, horizontally-transmitted life modes

135 of EHB found in diverse Dikarya (Araldi-Brondolo et al., 2017). Unlike Mycetohabitans spp.,

136 Luteibacter sp. 9143 does not have a T3SS, but does have type I, II, IV, and VI secretion systems

137 (Baltrus et al., 2017). Luteibacter sp. 9143 has not been observed to be vertically transmitted in

138 its host (i.e., it is not observed readily in conidia). It can be isolated reliably in culture (Arendt et

139 al., 2016). Luteibacter sp. 9143 increases the ability of Pestalotiopsis sp. 9143 to establish as an

140 endophyte in plant hosts (Araldi-Brondolo et al., 2017) and enhances its production of indole-3-

141 acetic acid (Hoffman et al., 2013). In addition, Pestalotiopsis sp. 9143 harboring Luteibacter sp.

1429143 exhibits increased cellulase activity, growth on cellulose-enriched growth medium, and

143 degradation of senescent leaf tissue (Araldi-Brondolo et al., 2017). 
In this study, we contextualized phenotypic observations by using a transcriptomic

145 approach to consider gene expression of Pestalotiopsis sp. 9143 and its facultative EHB

146 Luteibacter sp. 9143, when grown axenically and in co-culture. We used transcripts from axenic

147 cultures of the fungus to optimize assembly and annotation of its genome, and used that modified

148 assembly to map fungal transcripts in parallel to mapping bacterial transcripts to a previously

149 published genome sequence for Luteibacter sp. 9143 (Baltrus et al., 2017). Our study was

150 designed to test three main predictions. In previous phenotypic assays, we observed that

151 Luteibacter sp. 9143 emerges from its fungal host under conditions of stress, and grows readily

152 in a free-living state provided there is sulfur source besides inorganic sulfate (Arendt, 2015;

153 Arendt et al., 2016; Baltrus and Arnold, 2017). Therefore, we predicted that the bacterium would

154 be mildly parasitic on the fungal partner, as illustrated by enhanced bacterial growth but reduced

155 fungal growth in co-culture relative to the axenic state. In such a situation we would expect

156 upregulation of genes associated with bacterial growth in co-culture and changes in metabolic

157 genes of both partners related to molecular exchange of sulfur compounds or other metabolites.

158 Second, we predicted that genes upregulated in co-culture would reflect symbiosis-relevant

159 genes, especially if clustered or relevant to secretion systems or nutrient processing, and

160 coinciding with downregulation of genes associated with motility. In the fungus we would expect

161 upregulation of cellular repair mechanisms or other responses to infection, and changes to

162 secondary metabolite genes relevant to symbiotic establishment, such as upregulation of putative

163 signaling molecules. Third, we predicted transcriptional changes related to carbohydrates such as

164 cellulose, based on observed differences in cellulase activity of Pestalotiopsis sp. 9143 with and

165 without Luteibacter (Araldi-Brondolo et al., 2017). To address these predictions, we performed

166 RNA-seq and analysis of differential gene expression comparing Luteibacter sp. 9143 and 
167 Pestalotiopsis sp. 9143 grown together in co-coculture vs. separately (axenically). Here, we

168 report on the genome of Pestalotiopsis sp. 9143 as well as the results of our analysis of

169 differential gene expression.

170

\section{RESULTS}

Report of the genome of Pestalotiopsis sp. 9143. We generated and assembled the genome sequence of Pestalotiopsis sp. 9143 grown axenically and following antibiotic treatment

174 (GenBank: JAHZSN000000000.1) by hybrid assembly of Illumina and Oxford Nanopore reads

175 (see Methods). The final genome assembly was 46.3 mega base pairs (Mb) with 13,076 predicted

176 protein coding genes and 247 tRNAs (Table 1). The BUSCO score, an assessment of genome 177 completeness, was $94.6 \%$ representing 1,255 complete genes out of 1,312 (Table S1) in the

178 Dikarya subkingdom data set (Seppey et al., 2019). Profiling of secondary metabolite clusters

179 using antiSMASH (Blin et al., 2019) revealed 64 secondary metabolite regions, four of which

180 included neighboring protoclusters (Table S2).

\section{Bacterial density and total biomass of experimental cultures supports mild}

182 parasitism of Luteibacter on Pestalotiopsis. Prior to extracting RNA for analysis of differential

183 gene expression, we examined the growth of Luteibacter sp. 9143 and Pestalotiopsis sp. 9143

184 grown together in co-coculture as well as each grown axenically. The bacterium was not

185 observed in the axenic fungal culture, nor was the fungus present in the axenic bacterial culture.

186 The average density of free-living Luteibacter sp. 9143 (i.e., cells not associated with fungal

187 mycelium) in co-culture flasks was significantly greater than that in axenic culture flasks (Welch

$188 t$-test, $t=6.7, d f=2.2, p$-value $=0.02)($ Fig. 1A). The average density of free-living Luteibacter

189 sp. 9143 in co-culture flasks was $8.67 \times 10^{5} \pm 2.00 \times 10^{5} \mathrm{CFU} / \mathrm{mL}$, and that of Luteibacter sp. 
1909143 in axenic culture flasks was $6.67 \times 10^{4} \pm 4.62 \times 10^{4} \mathrm{CFU} / \mathrm{mL}$. The average dried biomass of

191 Luteibacter sp. 9143 in axenic bacterial culture flasks was $3.3 \pm 0.0 \mathrm{mg}$. In contrast, we observed

192 significantly reduced growth of Pestalotiopsis sp. 9143 in co-culture relative to axenic culture.

193 The average combined biomass of Pestalotiopsis sp. 9143 and Luteibacter sp. 9143 in co-culture

194 flasks was $43.3 \pm 0.01 \mathrm{mg}$, significantly less than $103.00 \pm 0.02 \mathrm{mg}$ of Pestalotiopsis sp. 9143 in

195 axenic culture flasks (Welch $t$-test, $t=5.0, d f=3.1, p$-value $=0.01)($ Fig. 1B).

Transcriptional changes in Luteibacter sp. 9143 during co-culture reflect increased

197 growth and a symbiotic response. During co-culture with Pestalotiopsis sp. 9143, Luteibacter

198 sp. 9143 upregulated 217 genes and downregulated 241 genes relative to its gene expression in

199 the axenic state (i.e., for an adjusted $p$-value or false discovery rate [FDR] of 0.01) (Fig. 2 and

200 Table S3). Our analysis of enrichment of gene ontology (GO) terms was largely uninformative,

201 as nearly all terms identified as upregulated were also identified as downregulated. (Fig. S1). We

202 observed that 37 of $54(69 \%)$ genes predicted to code for ribosomal proteins were upregulated

203 with a $\log _{2}$ fold change (L2FC) of $\sim 1.5-3.0$ (Table S3), which we speculate reflects enhanced

204 bacterial growth in co-culture (Fig. 1A). Twenty-eight of the 37 (76\%) upregulated ribosomal

205 protein genes are encoded in a cluster with four additional ribosomal protein genes not

206 differentially expressed (DE), whereas the remaining nine are scattered throughout the genome

207 (Table S3).

Luteibacter upregulates type VI secretion, signaling, and transport in co-culture.

209 Genes more strongly upregulated than the majority of the ribosomal proteins included those

210 involved in the type VI secretion system (T6SS). Most known for antibacterial activity, T6SSs

211 serve various roles including metal scavenging, biofilm formation, and interactions with

212 eukaryotic predators and hosts (Bernal et al., 2018; Bayer-Santos et al., 2019). In total, 11 of 13 
213 (85\%) genes predicted to code for T6SS proteins were upregulated, roughly $24 \%$ of the 46 genes

214 observed to be significantly and highly upregulated (i.e., adjusted $p$-value $\leq 0.01, \mathrm{~L} 2 \mathrm{FC} \geq 3.0$;

215 Fig. 3 and Table S3). Similarly, we detected upregulation of two hypothetical proteins within the 216 cluster that are likely co-regulated (Fig. 3 and Table S3).

Additionally, the most- and sixth-most-upregulated genes were also clustered, and were

218 predicted to be a nucleoside-diphosphate-sugar epimerase and a two-component sensor histidine

219 kinase, respectively (Table S3). Notably, the response regulator in this cluster is also upregulated

220 although it does not meet our FDR threshold for significance (L2FC $=2.8$; $p$-value $=0.02$ ). Two-

221 component regulatory systems composed of kinases and response regulators are key signal

222 transducers for detection of environmental or cellular changes by bacteria.

We also observed all (4/4) genes predicted to be associated with sorbitol/mannitol

224 transport that form a cluster, as well as one adjacent gene predicted to code for a mannitol

225 dehydrogenase, to be upregulated (Table S3). Similarly, five of 15 (33\%) genes predicted to

226 code for efflux pumps were upregulated.

228 observed many downregulated genes to be associated with type IV pilus assembly, chemotaxis,

229 and motility (Fig. 2). Although all 12 genes predicted to code for type IV secretion system

230 (T4SS) proteins were not DE, eight of 24 type IV pilus assembly genes were DE, and nearly all

231 (7/8 or $88 \%$ ) were downregulated (i.e., pilA, pilB, pilC, pilQ, pilV, and pilW). Only one gene,

232 pilY1, was upregulated. For the operon consisting of pilM, pilN, pilO, pilP, and pilQ, only pilQ is

233 downregulated.

234 Whereas several DE genes predicted to be associated with chemotaxis, flagella, and

235 motility are scattered throughout the genome of Luteibacter sp. 9143, the majority were 
organized in distinct clusters. For example, we found 18 of 41 genes (44\%) predicted to code for

237 flagellar proteins and eight of 34 genes (24\%) predicted to code for chemotaxis proteins to be

238 downregulated. This included genes predicted to code for the sigma-54 specific transcriptional

239 regulator FliA and an anti-sigma-28 factor in the FlgM family, which are two proteins important

240 for regulating the flagellar protein-coding genes (Osterman et al. 2015) (Table S3). Along with

24115 of the 18 downregulated genes coding for flagellar proteins and three downregulated genes

242 coding for chemotaxis, these genes form a cluster comprising 47 genes related to chemotaxis and

243 motility (Table S3).

244 Luteibacter downregulates methionine metabolism in co-culture. When considering

245 amino acid metabolism, we observed genes related to methionine to be DE. For example, all

246 (7/7) genes predicted to code for methionine metabolism or transport were downregulated. This

247 included a methionine aminotransferase, a methionine synthase, a methionine

248 adenosyltransferase, two peptide-methionine $\mathrm{S}$-oxide reductases ( $\mathrm{R}$ and $\mathrm{S}$ ), a methionine

249 transport system substrate-binding protein, and a homoserine $O$-acetyletransferase, the latter of

250 which was the most downregulated gene in the experiment (Table S3). Furthermore, three of

251 four genes involved in leucine biosynthesis and one gene involved in leucine degradation were

252 also downregulated, including the small and large subunits of 3-isopropylmalate dehydratase, 3-

253 isopropylmalate dehydrogenase, and 2-isopropylmalate synthase, and leucine dehydrogenase

254 (Fig. 2). This suggests an undescribed role for leucine or other branched chain amino acids

255 during the symbiosis.

256 Transcriptional changes in Pestalotiopsis sp. 9143 during co-culture may be

257 facilitated by NmrA-like transcription repressors. During co-culture with Luteibacter sp.

258 9143, Pestalotiopsis sp. 9143 upregulated 478 genes and downregulated 521 genes for an FDR 
of 0.01 (Fig. 4A and Table S4). In contrast to the bacterial genome, the limited number of gene

260 name annotations in the fungal genome made GO analysis a useful tool to identify changes in the

261 fungal transcriptome. There were $21 \mathrm{GO}$ terms enriched in the upregulated genes, and $22 \mathrm{GO}$

262 terms enriched in the downregulated genes (Fig. 4B). The GO terms with the highest enrichment

263 score among upregulated genes were the molecular function (MF) GO terms for ATPase activity

264 (GO:0016887) and ATPase coupled transmembrane transporter activity (GO:0042626). The top

265 biological processes (BP) GO terms were peptide pheromone export (GO:0000770) and

266 methionine metabolic process (GO:0006555), while the three enriched cellular component (CC)

267 GO terms were myosin complex (GO:0016459), integral component of the membrane

268 (GO:0016021), and microtubule (GO:0005874). The enriched GO terms in the downregulated

269 genes had lower enrichment scores overall, with the largest ones being vitamin B6 biosynthetic

270 process (GO:0042819; BP), membrane (GO:0016020; CC), flavin adenine dinucleotide binding

271 (GO:0050660; MF), and oxidoreductase activity, acting on $\mathrm{CH}-\mathrm{OH}$ group of donors

272 (GO:0016614; MF).

273 The top two upregulated genes (KJ359_005267 and KJ359_006927) both encode

274 hypothetical proteins with NAD(P)-binding domains that are members of the PFAM NmrA

275 (PF05368), suggesting they are transcription repressors (Andrianopoulos et al., 1998). Five

276 additional genes encoding NmrA-like proteins were upregulated, and another one was the ninth

277 most downregulated gene. While a handful of the most upregulated genes are cytochrome P450s

278 and there are some that are downregulated, these are not enriched in the data set as only 14 are

279 differentially expressed out of 234 total. Nearly half of the most downregulated genes (i.e., 13 of

$28030 ; \mathrm{L} 2 \mathrm{FC} \geq 6$ ) are hypothetical proteins with no annotated domains of known function. The

281 following sections detail the themes present in the differentially expressed genes and GO term 
282

283

284

285

286

287

288

289

290

291

292

293 294 (Reza et al., 2016).

295

296

297

298

299

300

301

302

303

enrichment, highlighting the changes in transporters and cell structure-related genes, methionine and carbohydrate metabolism, and potential defense or signaling compounds like secondary metabolites and $\beta$-lactamases.

Diverse transporters are up- and downregulated by Pestalotiopsis. Transport changes in Pestalotiopsis sp. 9143 are considerable in the presence of Luteibacter sp. 9143. The term for transmembrane transport (GO:0055085) is enriched in 42 up- and 31downregulated genes that are largely predicted to encode ATP-binding cassette (ABC) transporters or Major Facilitator Superfamily (MFS) transporters, as well as amino acid permeases, oligopeptide transporters, and metal ion transporters. Of the non-MFS and ABC protein-encoding genes, two purine-cytosine permeases (InterPro: IPR001248) are downregulated. Additionally, three genes putatively encoding for magnesium $\left(\mathrm{Mg}^{2+}\right)$ CorA-like transporter proteins are all downregulated, possibly reducing $\mathrm{Mg}^{2+}$ ion concentration and contributing to a reduction in growth and cell wall integrity

Among the downregulated genes associated with transmembrane transport, the majority are MFS transporters (21 of 31) and of those, 11 are from the sugar transporter MFS subfamily (IPR005828 and/or PF00083). In contrast, only five predicted sugar transporters are upregulated including the hexose transporter $h x t 1$. Among the genes that were upregulated, $19 \mathrm{ABC}$ transporters contributed to the most enriched GO terms: ATPase-coupled transmembrane transporter (GO:0042646), ATPase activity (GO:0016887), and ATP binding (GO:0005524).

Seven of the 19 are associated with peptide pheromone export (GO:0000770) and only two are predicted to be within secondary metabolite clusters: KJ359_000853 in Cluster 10.1 is upregulated and KJ359_002926 in Cluster 18.2 is downregulated, as mentioned detailed below. 
304 Thus, the substrates of many of these ABC transporters are unknown given the wide variety of

305 potential substrates ranging from lipids to toxins (Perlin et al., 2014).

307 manipulation of actin is common in close symbioses, such as rhizobial nodule formation in plant

308 roots (Zhang et al., 2019) and intracellular bacterial pathogen motility in animals (Goldberg,

309 2001), we predicted that cell wall- and membrane-associated genes would be differentially

310 expressed. We found that the upregulated genes are enriched for many GO terms associated with

311 the cytoskeleton and cell wall: chitin metabolic process (GO:0006030), myosin complex

312 (GO:0016459), microtubule (GO:0005874), motor activity (GO:0003774), actin binding

313 (GO:0003779), structural constituent of cytoskeleton (GO:0005200), and mannan endo-1,6-

314 alpha-mannosidase activity (GO:0008496). The genes associated with these terms (Table 2) are

315 mostly myosins, chitin synthases, tubulins, and mannan endo-1,6-alpha-mannosidases. Of the

316 three DE mannan endo-1,6-alpha-mannosidases, which are typically required for fungal cell

317 growth and contribute to fungal cell wall biosynthesis (Kitagaki et al., 2002), one is actually

318 downregulated. The DE genes of all other enriched GO terms associated with the cytoskeleton or

319 cell wall are upregulated (Table 2). These results align with the changes in gene expression seen

320 in other bacterial-fungal symbioses; a compatible Rhizopus microsporus isolate upregulates

321 genes involved in cytoskeletal rearrangement and the cell wall when in contact with

322 Mycetohabitans spp. (Lastovetsky et al., 2020).

323 Notably, many of the genes related to cell structure that were not considered differentially

324 expressed for our set FDR $<0.01$ are just beyond that probability threshold and typically have low

325 L2FCs. For example, of the five genes in the Pestalotiopsis sp. 9143 genome annotated with the

326 term myosin complex (GO:0016459), three genes are DE with a L2FC of 1.1-1.2 for an 
327 FDR $<0.01$, but the other two genes are DE for a less stringent FDR $<0.05$. In filamentous fungi,

328 myosins are associated with proper septation within hyphae, sporulation, and cell wall formation

329 (Guo et al., 2017b; Renshaw et al., 2016). Additionally, three of the six genes annotated with the

330 MARVEL domain (IPR008253) are downregulated (KJ359_003932, KJ359_001504, and

331 KJ359_002564) for FDR<0.01, whereas two of the remaining three have an FDR $<0.06$. Proteins

332 containing the MARVEL domain are not well studied in fungi but have been shown to play a

333 role in actin and membrane organization in Candida albicans (Douglas et al., 2013) and in cell

334 growth and fusion in Neurospora crassa (Fu et al., 2011).

Methionine metabolism is upregulated by Pestalotiopsis, complementing

336 downregulation by Luteibacter. Within the upregulated genes, the second most significantly

337 enriched GO term for biological processes is that for methionine metabolic process

338 (GO:0006555) (Fig. 4B). Three of 14 genes with this term or child GO terms are slightly

339 upregulated with a L2FC ranging from one to two: two predicted methylenetetrahydrofolate

340 reductases (KJ359_010927 and KJ359_003580) and one methionine synthase (KJ359_000681).

341 Some proteins involved in methionine metabolism use pyridoxal-5'-phosphate (PLP) as a

342 cofactor. However, two genes encoding PLP synthase subunits Snz1 (KJ359_006612) and

343 PdxT/SNO (KJ359_006611) are downregulated, along with a putative PLP oxidase

344 (KJ359_005893). As PLP is a vitamin B6 compound, these genes represent the enrichment of the

345 term for vitamin B6 biosynthetic process (GO:0042819) among the downregulated genes.

346 Glycoside hydrolases account for most changes in Pestalotiopsis carbohydrate

347 metabolism during co-culture. We previously observed that cured Pestalotiopsis sp. 9143 has

348 reduced cellulase activity and reduced growth on cellulose-based media (Araldi-Brondolo et al.,

349 2017), thus we expected to see changes in carbohydrate metabolism when co-cultured. The 
majority of the downregulated genes (16 of 23) predicted to be involved in the carbohydrate

351 metabolic process (GO:0005975) encode putative members of glycoside hydrolase families;

352 altogether 15 upregulated and 21 downregulated genes are predicted by InterPro to encode

353 glycoside hydrolases. Glycoside hydrolases act on diverse substrates and are commonly secreted

354 out of the fungal cell to break down biomass, including compounds found in plant cell walls like

355 lignin, cellulose, and hemicellulose (Murphy et al., 2011). Of the DE glycoside hydrolases, 25

356 have signal peptides predicted by SignalP (Almagro Armenteros et al., 2019).

Two downregulated genes involved in the carbohydrate metabolic process (GO:0005975)

358 are also annotated with carbohydrate phosphorylation (GO:0046835) and encode hexokinases.

359 The additional carbohydrate metabolism-associated genes are a glucosamine-6-phosphate

360 isomerase, a sugar phosphate isomerase (RpiB/LacA/LacB family), and a polysaccharide

361 deacetylase. The glucosamine-6-phosphate isomerase (KJ359_003080) converts glucosamine-6-

362 phosphate to fructose-6-phosphate and ammonium in the last step of $\mathrm{N}$-acetylglucosamine

363 (GlcNAC) catabolism.

Only secondary metabolite Cluster 10.1 is fully differentially expressed in co-culture.

365 Of the 64 secondary metabolite clusters (four of which include neighboring clusters) in the

366 Pestalotiopsis sp. 9143 genome, 13 have a core biosynthetic gene (i.e., NRPS, PKS, NRPS-PKS

367 hybrid, or terpene cyclase) that is DE when co-cultured with Luteibacter sp. 9143 (Fig. S2). Six

368 were downregulated and five were upregulated. However, only in Cluster 10.1 are the core PKS

369 genes and neighboring biosynthetic and transport genes all DE (Fig. 5A, B, and Fig. S2). Cluster

37018.2 has a DE core NRPS (KJ359_002925) and ABC transporter gene (KJ359_002926), but

371 they are downregulated and the additional $\alpha / \beta$ hydrolase (KJ359_002922) predicted to be

372 involved in biosynthesis is not DE (Fig. 5A, C, and Fig. S2). 
Cluster 10.1 contains genes putatively encoding two polyketide synthases (PKS), two

374 glycosyltransferases, a cytochrome P450, and an ABC transporter (Fig. 5B). Additional genes

375 for an acyltransferase and an amidohydrolase were not predicted as serving a role in the cluster,

376 but are upregulated and may also contribute to a final product. The eight genes in total that were

377 upregulated in Cluster 10.1 ranged from a L2FC of 2.5 to 7. Both predicted PKS proteins

378 encoded by KJ359_00848 and KJ359_00851 have the required ketosynthase (KS),

379 acyltransferase (AT), and phosphopanetheine (PP) acyl carrier protein (ACP) group domains.

380 KJ359_00848 has a carbon methyltransferase domain (cMT) and three additional reducing

381 domains: dehydratase (DH), enoylreductase (ER), and ketoreductase (KR). In contrast,

382 KJ359_00851 has a product template (PT) domain and a N-terminal ACP transacylase similar to

383 the one involved in aflatoxin biosynthesis (SAT).

Differentially expressed $\beta$-lactamases play an unknown role in fungi. Five $\beta$-lactamase-

385 related (IPR001466) genes are upregulated $(1.7<\mathrm{L} 2 \mathrm{FC}<7.1)$ while one is downregulated

$386(\mathrm{~L} 2 \mathrm{FC}=-4.0)$. In bacteria, $\beta$-lactamases confer antibiotic resistance against $\beta$-lactams which are

387 produced by fungi to target bacterial peptidoglycan, with few examples of fungicidal $\beta$-lactams

388 (Arnoldi et al., 1990). Yet, there are few $\beta$-lactamase-related genes characterized in fungi so the

389 computational annotation of $\beta$-lactamases in fungal genomes is likely too specific and does not

390 account for potential diversity of lactamases (Gao et al., 2017).

\section{DISCUSSION}

We used an RNA-seq experiment to understand the transcriptional responses of the EHB

394 Luteibacter sp. 9143 and foliar endophyte Pestalotiopsis sp. 9143 when grown together in co-

395 culture vs. axenically. Based on previous experiments and preliminary data, we predicted that the 
bacterium would be mildly parasitic on the fungus, and would respond to co-culture with its host

by altering transcription of genes related to chemotaxis, motility, nutrient acquisition, and

secretion systems. Similarly, for the fungus, we predicted changes related to carbohydrates such

as cellulose and the upregulation of genes related to repair mechanisms and responses to

infection related to symbiosis with Luteibacter. These predictions were upheld by our analyses.

bacterial culture. This supports previous findings that the bacterium is not able to obtain sulfur in

404 the form of sulfate when grown in pure culture, and that growing in co-culture with its host

405 fungus can supplement this deficiency (Baltrus and Arnold, 2017). Upregulation of genes

406 encoding ribosomal proteins during co-culture was consistent with the increased bacterial

407 growth. At the same time, we observed a greater biomass of Pestalotiopsis sp. 9143 grown in

408 pure culture compared to that of the fungus growing with its EHB in co-culture, implying that

409 fungal growth is inhibited in the presence of Luteibacter sp. 9143. Whether or not this reduction

410 in growth of the fungus in the presence of the bacterium influences interactions with host plants

411 remains unknown, but should be explored further.

Given the reduction in growth by Pestalotiopsis sp. 9143 in co-culture, the fungus may be

413 undergoing a defense response to Luteibacter sp. 9143. Indicators of this include the

414 upregulation of diverse transporters and $\beta$-lactamase genes, which could be turned on in response

415 to or anticipation of antifungal compounds. For example, a Fusarium verticillioides "metallo- $\beta$ -

416 lactamase" protein actually confers resistance to plant-derived, antifungal $\gamma$-lactams (Glenn $e t$

$417 a l ., 2016)$. Similarly, the upregulated lactamase genes in Pestalotiopsis sp. 9143 may play a role

418 in countering antifungal compounds or in improving the environment for putative bacterial 
partners. Furthermore, it is likely at least a subset of the upregulated transporters mediate resistance to antifungal compounds, especially four differentially expressed genes (KJ359_009703, KJ359_010943, KJ359_010406, and KJ359_003031) that have conserved

422 domains from the pleiotropic drug resistance protein family (PF06422). Antifungal resistance-

423 related gene expression may be in response to Luteibacter sp. 9143 specifically or bacteria

424 generally, and may be fruitful avenues to explore for host specificity in bacterial-fungal 425 interactions.

The response of Luteibacter to Pestalotiopsis in co-culture includes bacterial cells in

427 multiple stages of interaction, including free-living, attached, entering, and endohyphal,

428 complicating interpretation of the transcriptional response. This may contribute to why we see

429 low L2FC for cell wall- and membrane-associated genes in the fungus, as the majority of

430 bacterial cells are likely external to the fungus. Still, we see the response of Luteibacter sp. 9143

431 in co-culture with Pestalotiopsis sp. 9143 as representing an immediate metabolic response to

432 changes in environmental conditions and the initiation of association with the fungal partner.

433 This includes the downregulation of methionine metabolism, chemotaxis, and motility, and the

434 upregulation of sorbitol and mannitol transport, and T6SS (Fig. 2, 3). In particular, we speculate

435 that the upregulation of the T6SS may help Luteibacter to initiate and establish symbiosis with

436 Pestalotiopsis, although recognize that the bacterium may be using the T6SS for other purposes

437 such as nutrient acquisition or even defense (Cianfanelli et al., 2016; Si et al., 2016; Bayer-

438 Santos et al., 2019). Whereas it is difficult to understand which metabolites may be exchanged

439 during this interaction from these genomic data, future studies using metabolomics may provide

440 additional insight (e.g., Spraker et al., 2016). 
442 the establishment of fungal-bacterial partnerships, including both recruitment and invasion. In

443 the R. microsporus and Mycetohabitans interaction, secondary metabolites are not thought to

444 play a large role, as only one NRPS is upregulated and one PKS downregulated in $R$.

445 microsporus (i.e., the natural host strain) when in contact with the bacterium (Lastovetsky et al.,

446 2020). In contrast the Ralstonia solanacearum lipopeptide, ralsolamycin, induced substantial

447 developmental shifts in host fungi, enhancing bacterial entry into fungal chlamydospores

448 (Spraker et al., 2016). In our analysis, Pestalotiopsis differentially expresses the core

449 biosynthetic gene in 13 secondary metabolite clusters, but only Cluster 10.1 had all predicted

450 biosynthetic genes differentially expressed. Given the two PKS genes in Cluster 10.1, it is

451 possible that two separate metabolites are made, but the co-regulation and tight clustering

452 support a single metabolic pathway. For example, a single Aspergillus nidulans cluster creates

453 asperfuranone using two PKS genes, one highly reducing and the other not, along with five

454 additional genes (Chiang et al., 2009). Cluster 10.1 does not appear to be related to the

455 asperfuranone cluster based on the difference in accessory gene content and lack of homology

456 between KJ359_00848 and KJ359_00851 and the asperfuranone PKS genes, though it does

457 similarly contain one PKS predicted to be highly reducing and one not. Notably, this cluster is

458 present in Neopestalotiopsis clavispora, Neopestalotiopsis sp. 37M, and Pestalotiopsis

459 microspora at nearly $80 \%$ protein identity, suggesting conservation in closely related fungi. We

460 also observed Luteibacter sp. 9143 to upregulate genes predicted to code for multidrug efflux

461 pumps, which may be in response to the production of secondary metabolites by Pestalotiopsis

462 sp. 9143 and assist the bacterium in colonizing its host, especially if the fungus is producing

463 them as a defense response to bacterial invasion. 
Our study supports a more direct exchange of primary metabolites, as Luteibacter sp.

9143 cannot utilize the sulfate in minimal media as a sulfur source, thus requiring methionine or

466 other organic sulfur compounds generated by the fungus (Baltrus and Arnold, 2017).

467 Methionine is limited in the plant apoplast and methionine synthases are critical in plant

468 pathogenic ascomycetes for survival in planta (Solomon et al., 2000; Saint-Macary et al., 2015).

469 The small upregulation of methionine synthesis genes by the fungus may indicate a feedback

470 response to having sulfur-containing compounds be depleted by the presence of Luteibacter sp.

4719143 , or may be more actively encouraged by the bacterium in some way. In Saccharomyces

472 cerevisiae, excess methionine is tied to upregulation of synthesis of the cofactor pyridoxal-5'-

473 phosphate (PLP) (Walvekar \& Laxman, 2019), as part of an anabolic program leading to

474 increased synthesis of amino acids. The downregulation of PLP synthases in Pestalotiopsis sp.

4759143 could be tied to decreased methionine availability based on bacterial use, potentially

476 leading to less amino acid metabolism and contributing to the observed growth restriction.

477 Interestingly, genes predicted to be associated with methionine metabolism were downregulated

478 in Luteibacter sp. 9143 (Fig. 2B), which also supports the hypothesis that the bacterium is

479 acquiring methionine from Pestalotiopsis sp. 9143.

Altogether, our transcriptomic analysis supports the phenotypic findings we have

481 observed in the Pestalotiopsis-Luteibacter partnership, including a dependence on the fungal

482 host as a sulfur source for Luteibacter, a change in extracellular carbohydrate metabolism by

483 Pestalotiopsis when Luteibacter is present, and inverse growth impacts on the partners during

484 co-culture. Together, these findings support a working model in which Luteibacter switches

485 from a motile to sedentary lifestyle in association with the fungus, which can provide it with

486 methionine as a sulfur source. Other metabolites such as leucine, mannitol, or sorbitol may also 
487 be exchanged. The specific metabolites and signaling pathways leading to the transcriptional

488 changes remain to be investigated, but may involve the highly upregulated bacterial two-

489 component system or T6SS and the fungal PKS secondary metabolite Cluster 10.1 or NmrA

490 repressors. Based on the diversity of EHB-fungal relationships, additional work to probe the

491 mechanisms underlying this and other partnerships will be critical for informing a broader model

492 of bacterial-fungal interactions, and especially how they relate to ecology in the phyllosphere.

494 MATERIALS AND METHODS

495 We obtained Pestalotiopsis sp. 9143 from a living culture collection at the Robert L.

496 Gilbertson Mycological Herbarium, University of Arizona, Tucson (ARIZ). The fungus was

497 isolated originally as an endophyte from healthy, asymptomatic foliage of Platycladus orientalis

498 (Cupressaceae; Hoffman and Arnold, 2008). Pestalotiopsis sp. 9143 was naturally infected with

499 the endohyphal bacterium Luteibacter sp. 9143 at the time of isolation, and maintained a

500 consistent infection throughout growth in culture on various media and vouchering in sterile

501 water. The bacterium was isolated successfully in culture and a rifampicin-resistant strain (9143)

502 was generated by plating on LB amended with $50 \mu \mathrm{g} / \mathrm{mL}$ rifampicin (Arendt et al., 2017).

To generate cured Pestalotiopsis sp. 9143, conidia from sporulating, 21 day-old cultures

504 were transferred to a new Petri plate $(60-\mathrm{mm})$ containing $2 \%$ malt extract agar MEA amended

505 with four antibiotics: tetracycline $(10 \mu \mathrm{g} / \mathrm{mL})$, ampicillin $(100 \mu \mathrm{g} / \mathrm{mL})$, ciprofloxacin $(40 \mu \mathrm{g} / \mathrm{mL})$

506 and kanamycin $(50 \mu \mathrm{g} / \mathrm{mL} ; \mathrm{MEA}+\mathrm{TACK})$. Total genomic DNA extracted from fungal mycelium

507 was used for polymerase chain reaction (PCR) to screen for presence or absence of Luteibacter

508 sp. 9143 by amplifying the bacterial $16 S$ ribosomal RNA (rRNA) gene following (Shaffer et al., 
2016). Successful amplification was assessed by mixing PCR products with SYBR green and

510 running on a $2 \%$ agarose gel in Tris-EDTA buffer (1X).

512 Luteibacter sp. 9143 in Pestalotiopsis sp. 9143 growing on MEA+TACK, conidia were

513 transferred to a new MEA plate. We then revived Luteibacter sp. 9143 by streaking from

514 glycerol stock onto lysogeny broth (LB) agar (1\% NaCl; i.e., LB-Miller). Both fungal and

515 bacterial cultures were incubated at ambient laboratory conditions. After 21 days, for

516 Pestalotiopsis sp. 9143, six ca. 2- $\mathrm{mm}^{2}$ squares were excised from the growing edge of the fungal

517 colony with a sterile toothpick, and transferred to a sterile $125-\mathrm{mL}$ flask containing $50 \mathrm{~mL}$ fresh

518 liquid M9 + glucose $(2 \%)+$ methionine $(10 \mathrm{mM})$ (high-methionine minimal) medium. In parallel

519 for Luteibacter sp. 9143, a sterile toothpick was used to transfer bacterial cells from a single

520 colony to a test tube containing $5 \mathrm{~mL}$ fresh liquid high-methionine minimal medium. Fungal and

521 bacterial cultures were then incubated at $27^{\circ} \mathrm{C}$ shaking at 200 rotations per minute (rpm).

After 10 days we processed cultures to produce one culture flask for each of the

523 following treatments: (1) Pestalotiopsis sp. 9143 grown alone (i.e., axenic fungus); (2)

524 Luteibacter sp. 9143 grown alone (i.e., axenic bacterium); and (3) Pestalotiopsis sp. 9143 and

525 Luteibacter sp. 9143 grown together (i.e., fungus + bacterium co-culture). Fungal inoculum was

526 created by transferring mycelia from the fungal culture flask to a sterile 50 -mL stainless steel

527 Sorvall omni mixer homogenizer chamber assembly (5.08-cm blade, PTFE bearings; OMNI,

528 Kennesaw, GA, USA) containing $15 \mathrm{~mL}$ fresh liquid high-methionine minimal medium for

529 homogenization: (20s on, 1 min off, 20s on). Bacterial inoculum was created by diluting the

530 liquid culture to an optical density at 600 nanometers $(\mathrm{nm})\left(\mathrm{OD}_{600}\right)$ of 0.1 . Five milliliters of

531 mycelial suspension and of bacterial suspension were added to flasks with fresh liquid high- 
532 methionine minimal medium to create an axenic fungal, an axenic bacterial, and a fungal-

533 bacterial co-culture, each at a final volume of $50 \mathrm{~mL}$.

After four days we split cultures to produce three biological replicates for each of the

535 three treatments above. We first transferred the contents of each culture flask to a sterile 50-mL

536 Falcon tube (Corning, NY, USA), centrifuged at $11000 \mathrm{rpm}$ for $20 \mathrm{~min}$ (5430R, Eppendorf,

537 Hamburg, Germany) to pellet cells, removed media by pipetting, and re-suspended in fresh liquid

538 M9 + glucose $(2 \%)+$ methionine $(100 \mu \mathrm{M})$ (low-methionine minimal) medium to bring the total

539 volume of each tube to $25 \mathrm{~mL}$. We then transferred $5 \mathrm{~mL}$ of each culture to four sterile $125-\mathrm{mL}$

540 flask containing $45 \mathrm{~mL}$ fresh liquid low-methionine minimal medium and incubated all cultures

541 at $27^{\circ} \mathrm{C}$ shaking at $200 \mathrm{rpm}$. Previous work indicates Luteibacter sp. 9143 cannot utilize sulfate

542 as a sulfur source during laboratory growth, but is able to grow in media supplemented with

543 cysteine, methionine or high levels of thiosulfate, as well as in co-culture with its host fungus

544 Pestalotiopsis sp. 9143 (Baltrus and Arnold, 2017). Although we used a high methionine

545 medium in order to allow for robust growth of Luteibacter sp. 9143 up to this point, we now

546 reduce its concentration in hopes of inducing association of Luteibacter sp. 9143 with

547 Pestalotiopsis sp. 9143 in co-culture, as the bacterium appears to acquire sulfur from the host

548 fungus (Baltrus and Arnold, 2017).

549 Bacterial density and total biomass of experimental cultures. After three days and

550 immediately prior to RNA extraction, we quantified the density of free-living Luteibacter sp.

5519143 (i.e., those cells not associated with fungal mycelium in co-culture) in all cultures by

552 removing $50 \mu \mathrm{L}$ of liquid culture, diluting $1: 1 \mathrm{M}$, spreading $50 \mu \mathrm{L}$ onto the surface of a Petri

553 plate $(100-\mathrm{mm})$ containing LB agar, and incubating at $27^{\circ} \mathrm{C}$ for $48 \mathrm{~h}$ prior to counting colony

554 forming units (CFU). Following plating, we recovered and lyophilized all remaining tissue in 
each culture by first transferring to a 50-mL Falcon tube and centrifuging at $11000 \mathrm{rpm}$ for 20 bacteria associated with fungal mycelia, in order to prevent transcriptional responses (e.g., such as to washing), and note that the population of Luteibacter sp. 9143 in co-cultured flasks includes free-living, externally-associated, and endohyphal cells. We next transferred each pellet to a distinct, sterile 1.7-mL microcentrifuge tube with the lid punctured using a sterile needle, and immediately submerged samples in liquid nitrogen until boiling stops. We then transferred sample tubes to a pre-cooled lyophilizer flask and lyophilized samples for $24 \mathrm{~h}$ prior to storing at

$563-80^{\circ} \mathrm{C}$.

Extraction of RNA from experimental cultures. For axenic fungal- and co-cultured samples, we transferred lyophilized tissue to a pre-weighed, sterile 1.7-mL microcentrifuge tube, ground tissue using a sterile pestle, and obtained total biomass by weighing and subtracting the

567 weight of the tube. For axenic bacterial samples, we transferred $1.5 \mathrm{~mL}$ suspended cells to a sterile 1.7-mL microcentrifuge tube, pelleted cells, removed excess media, and flash froze before lyophilizing cells. To extract RNA, for each sample we used either $0.05 \mathrm{~g}$ tissue $(\mathrm{ca} .100 \mu \mathrm{L}$ of

570 ground tissue; axenic fungal- and co-cultured samples) or entire lyophilized cell pellets (axenic

571 bacterial samples). For each sample, biomass was added to a sterile 1.7-mL microcentrifuge tube 572 containing sterile zirconium oxide beads. We then added $1 \mathrm{~mL}$ TRIzol to each sample tube,

573 macerated samples using a beadbeater run for 2 min (power level 10), incubated samples on ice

574 for $20 \mathrm{~min}$, and centrifuged at $12000 \mathrm{rpm}$ for $15 \mathrm{~min}$ at $4^{\circ} \mathrm{C}$ (5804 Eppendorf) to pellet tissue.

575 We next transferred supernatants of each sample to sterile 1.7-mL microcentrifuge tube, added

$576250 \mu \mathrm{L}$ chloroform, and mixed all samples uniformly by placing in a single tube rack, shaking

577 the rack for $15 \mathrm{~s}$, allowing to rest for $15 \mathrm{~s}$, and shaking again for $15 \mathrm{~s}$. We then incubated 
578 samples at ambient laboratory conditions for $5 \mathrm{~min}$, centrifuged at maximum speed for $15 \mathrm{~min}$ at

$5794^{\circ} \mathrm{C}$ (5804 Eppendorf) to separate phases, transferred $400 \mu \mathrm{L}$ of the upper, aqueous layer of each

580 sample to a distinct, sterile $1.7-\mathrm{mL}$ microcentrifuge tube discarding the pellet, and added $750 \mu \mathrm{L}$

581 of pre-chilled $\left(-20^{\circ} \mathrm{C}\right)$ ethanol $(100 \%)$ to each sample tube. We inverted sample tubes several

582 times uniformly as above, and incubated them on ice for $10 \mathrm{~min}$. Nucleotides were observed

583 precipitating in each sample. We next centrifuged sample tubes at maximum speed for 15 min at

$5844^{\circ} \mathrm{C}$ as above, removed and discarded supernatant from each sample tube by pipetting, washed

585 each pellet with $1 \mathrm{~mL}$ ethanol (75\%), decanted the supernatant, and added $1 \mathrm{~mL}$ ethanol (75\%

586 with DEPC water) to each sample tube. We then centrifuged samples at $14000 \mathrm{rpm}$ for $5 \mathrm{~min}$ at

$5874^{\circ} \mathrm{C}$, decanted the supernatant, and dried pellets by centrifuging in a vacuufuge at $12000 \mathrm{rpm}$ for

$5883 \mathrm{~min}$ at $30^{\circ} \mathrm{C}$. We next re-suspended each pellet in $40 \mu \mathrm{L}$ DEPC water and incubated sample

589 tubes for $5 \mathrm{~min}$ at $65^{\circ} \mathrm{C}$, prior to storage at $-80^{\circ} \mathrm{C}$.

590 We quantified the 260/280 (i.e., nucleotide/protein) and 260/230 (nucleotide/organic)

591 ratios for each sample using a NanoDrop (pre-DNase ratios), prior to treatment with DNase

592 using the DNA-free kit (Ambion AM1906, ThermoFisher, Waltham, MA, USA). Briefly, for

593 each sample we transferred a volume equivalent to $20 \mu \mathrm{g}$ total nucleotide to a sterile $1.7-\mathrm{mL}$

594 microcentrifuge tube and added DEPC water to obtain a total volume of $45 \mu \mathrm{L}$. We then added

595 DNaseI Buffer to each sample, vortexed gently, added $1 \mu \mathrm{L}$ rDNase, vortexed gently, and

596 incubated sample tubes for $30 \mathrm{~min}$ at $35^{\circ} \mathrm{C}$. For all samples we then repeated adding $1 \mu \mathrm{L}$

597 rDNase, vortexing, and incubating for $30 \mathrm{~min}$ at $35^{\circ} \mathrm{C}$. We next added $5 \mu \mathrm{L}$ DNaseI Inactivation

598 Suspension to each sample tube and incubated at ambient laboratory conditions for 2 min, hand-

599 vortexing every 30 s. We quantified 260/280 and 260/230 ratios for each sample again as above

600 (post-DNase ratios), diluted each sample to a concentration of $400 \mathrm{ng} / \mu \mathrm{L}$ in DEPC water to 
601 produce a total volume of $50 \mu \mathrm{L}$, and submitted sample tubes to the University of Arizona

602 Genetics Core (UAGC) for quality checking, rRNA depletion and poly-A selection, and

603 sequencing.

604 RNA sequencing. From all fungal RNA samples, fungal rRNA was depleted twice with

605 the Ribominus Transcriptome Isolation Kit for yeast (Thermofisher) and poly-A selection to

606 acquire fungal transcripts was done using the NEBNext Poly(A) mRNA magnetic isolation

607 module (New England BioLabs). The remaining RNA from the co-cultured samples and the

608 RNA from the axenic bacteria samples were depleted twice of bacterial rRNA using the

609 Ribominus Transcriptome Isolation Kit for bacteria (Thermofisher). All subsequent quality

610 checking was performed by the University of Arizona Genomics Core (UAGC) using an Agilent

611 Bioanalyzer 2100 and RNA 6000 Pico Chips. Sequencing libraries were prepared using RNA

612 TruSeq library construction kits (Illumina, San Diego, CA, USA) and sequenced on an Illumina

613 HiSeq 2500 using TruSeq 2 x 100 base pair (bp) paired-end chemistry (Illumina) by UAGC.

Sequencing and assembly of Pestalotiopsis sp. 9143 genome. Genomic DNA of

615 Pestalotiopsis sp. 9143 was prepared using following Arnold and Lutzoni (2007). Genomic DNA

616 was sent to the Microbial Genome Sequencing Center (MiGS) for library preparation using an

617 Illumina tagmentation kit and paired-end (2 x $150 \mathrm{bp})$ sequencing with a NextSeq 550 instrument

618 as per Baym et al. (2015). Long-read sequencing was done in the Baltrus lab from the same

619 genomic DNA sample. DNA was prepared for sequencing using the LSK109 ligation

620 sequencing kit without shearing and using the Long Fragment Buffer. Reads were sequenced

621 using an R9.4 flowcell in a MinION, with basecalling by Guppy (v.3.2.6).

622 Illumina and Nanopore sequencing reads were used to create a de novo hybrid assembly

623 with MaSuRCA (v3.4.1) (Zimin et al., 2013; Zimin et al., 2017). The resulting assembled 
624 genome (Table S5) FASTA file was used as input for gene prediction and annotation by the

625 funannotate (v1.7.4) pipeline (Palmer \& Stajich, 2016). Repetitive contigs were removed and the 626 remaining contigs sorted before repeat masking. RNA-seq reads assembled with Trinity (Haas et 627 al., 2013) were used as transcript evidence for gene prediction of the softmasked genome.

628 Putative functional annotation by funannotate (Table S6) was informed by searches of UniProt 629 DB version 2020_04, antiSMASH 5.0 (Blin et al., 2019), Phobius (Käll et al., 2007),

630 InterproScan5 (Jones et al., 2014), dbCAN v9.0 (Yin et al., 2012), MEROPS v12.0 (Rawlings et 631 al., 2013) and eggNOG-mapper v1.0.3 (Huerta-Cepas et al., 2017). Two scaffolds were flagged 632 as part of the mitochondrial genome and annotated with MITOS (Bernt et al. 2013). The genome 633 assembly and annotations were deposited in NCBI Genomes (JAHZSN000000000.1) and the 634 raw reads were deposited in the Sequence Read Archive (SRR14629398; SRR14629399). All 635 sequencing from this study can be accessed through BioProject PRJNA732082.

Analysis of RNA-seq. Illumina data were quality controlled using QoRTs (Hartley and

637 Mullikin, 2015). We mapped fungal reads onto the genome of Pestalotiopsis sp. 9143

638 (JAHZSN000000000.1), and bacterial reads onto that of Luteibacter sp. 9143

639 (JQNL00000000.1; Baltrus et al., 2017) using STAR v2.7.3 (Dobin et al., 2013). Mapped reads

640 were subset with SAMtools (Li et al., 2009) and transcript abundances quantified using

641 featureCount, part of the subread (v2.0.1) package (Liao et al., 2014) (Tables S7, S8). One

642 axenic fungal sample had triple the reads from sequencing, so the reads were randomly

643 downsampled with SAMtools to be more closely aligned with the number of reads from the other

644 samples (Table S8). We carried out analysis of differential gene expression using the R package

645 DESeq2 (Love et al., 2014; R Core Team, 2021) with a false discovery rate (FDR) value of 0.01

646 as a threshold for differential expression. We looked for enrichment for specific gene ontology 
647 (GO) terms among all differentially expressed genes for both Pestalotiopsis sp. 9143 and

648 Luteibacter sp. 9143 (i.e., grown in co-culture vs. axenically). Separately for the fungus and the

649 bacterium, we first generated a list of differentially expressed genes and corresponding GO

650 annotations based on the reference genome, and then conducted a functional enrichment analysis

651 using a Fisher's Exact Test with a $p$-value of 0.01 (Conesa et al., 2005) using the R package

652 topGO (Alexa and Rahnenfuhrer, 2021). Raw sequencing reads and processed featureCounts

653 tables can be accessed through the NCBI Gene Expression Omnibus (GSE181155).

\section{AUTHOR CONTRIBUTIONS}

656 AEA and DAB conceived of the study, with JPS and JES. AEA provided the original fungal and

657 bacterial material. JPS and JES cultured the fungus and bacterium, cured the fungus of bacteria, 658 and conducted experiments; JPS, JES, and MEC analyzed data; KH and DB conducted

659 preliminary experiments crucial to the work; BS assisted with data analysis; MC assisted with 660 genomic DNA extraction for Pestalotiopsis; JPS, JES, MEC, DB, and AEA led the development 661 of the manuscript, with contributions from all authors.

\section{FUNDING}

664 We thank the National Science Foundation (NSF 1354219 to DB, AEA, and REG; NSF

665 postdoctoral research fellowship award no. 1612169 to JES, AEA; NSF-IGERT Fellowship to

666 JPS) and the School of Plant Sciences (Graduate Award to JPS) for supporting this work.

667 Additional support from the School of Plant Sciences and College of Agriculture and Life

668 Sciences at the University of Arizona is gratefully acknowledged. 


\section{ACKNOWLEDGMENTS}

671 An allocation of computer time from the UA Research Computing High Performance Computing

672 (HPC) at the University of Arizona is gratefully acknowledged.

673

674

675

676

677

678

679

680

681

682

683

684

685

686

687

688

689

690

691

692

693

694

695

696

697

698

699

700

701

702

703

704

705

706

707

708

709

\section{REFERENCES}

Alexa A, Rahnenfuhrer J. 2021. topGO: Enrichment analysis for gene oncology. R package version 2.44.0.

Almagro Armenteros JJ, Tsirigos KD, Sønderby CK, Petersen TN, Winther O, Brunak S, von Heijne G, Nielsen H. 2019. SignalP 5.0 improves signal peptide predictions using deep neural networks. Nat Biotechnol 37:420-423. doi: 10.1038/s41587-019-0036-z.

Andrianopoulos A, Kourambas S, Sharp JA, Davis MA, Hynes MJ. 1998. Characterization of the Aspergillus nidulans nmrA Gene Involved in Nitrogen Metabolite Repression. J Bacteriol 180:1973-1977. doi: 10.1128/JB.180.7.1973-1977.1998.

Araldi-Brondolo SJ, Spraker JE, Shaffer JP, Woytenko EH, Baltrus DA, Gallery RE, Arnold AE. 2017. Bacterial endosymbionts: Master modulators of fungal phenotypes. Microbiology Spectrum 5:5. doi: 10.1128/microbiolspec.FUNK-0056-2016.

Arendt KR. 2015. Master's Thesis. University of Arizona, Tucson, AZ. Symbiosis Establishment and Ecological Effects of Endohyphal Bacteria on Foliar Fungi.

Arendt, KR, Hockett KL, Araldi-Brondolo SJ, Baltrus DA, Arnold AE. 2016. Isolation of endohyphal bacteria from foliar Ascomycota and In Vitro establishment of their symbiotic associations. Appl Environ Microbiol 82:2943-2949. doi: 10.1128/AEM.00452-16.

Arnold, AE, Lutzoni F. 2007. Diversity and host range of foliar fungal endophytes: are tropical leaves biodiversity hotspots? Ecology 88:, 541-549. doi: 10.1890/05-1459.

Arnoldi A, Cabrini MR, Farina G, Merlini L. 1990. Activity of a series of beta-lactams against some phytopathogenic fungi. J Agric Food Chem 38:2197-2199. doi: 10.1021/jf00102a019.

Baltrus, DA, Arnold AE. 2017. Unusual Sulfur Requirements During Laboratory Growth of Luteibacter. bioRxiv. doi: 10.1101/149401.

Baltrus DA, Dougherty K, Arendt KR, Huntemann M, Clum A, Pillay M, Palaniappan K, Varghese N, Mikhailova N, Stamatis D, Reddy TBK, Ngan CY, Daum C, Shapiro N, Markowitz V, Ivanova N, Kyrpides N, Woyke T, Arnold AE. 2017. Absence of genome reduction in diverse, facultative endohyphal bacteria. Microbial Genomics 3:e000101. doi: 10.1099/mgen.0.000101.

Bayer-Santos E, Ceseti LM, Farah CS, Alvarez-Martinez CE. 2019. Distribution, Function and Regulation of Type 6 Secretion Systems of Xanthomonadales. Frontiers Microbiol 10:1635. doi: 10.3389/fmicb.2019.01635.

Baym M, Kryazhimskiy S, Lieberman TD, Chung H, Desai MM, Kishony R. 2015. Inexpensive multiplexed library preparation for megabase-sized genomes. PLoS One 10:e0128036. doi: 10.1371/journal.pone.0128036.

Bernal P, Llamas MA, Filloux A. 2018. Type VI secretion systems in plant-associated bacteria. Environ Microbiol 20:1-15. doi: 10.1111/1462-2920.13956. 
Bernt M, Donath A, Jühling F, Externbrink F, Florentz C, Fritzsch G, Pütz J, Middendorf M, Stadler PF. 2013. MITOS: Improved de novo metazoan mitochondrial genome annotation. Mol Phylogenet Evol 69:313-319. doi: 10.1016/j.ympev.2012.08.023. transmission of endobacteria in the arbuscular mycorrhizal fungus Gigaspora margarita through generation of vegetative spores. Appl Environ Microbiol 70:3600. doi: 10.1128/AEM.70.6.3600-3608.2004.

Bianciotto V, Lumini E, Bonfante P, and Vandamme P. 2003. Candidatus Glomeribacter gigasporarum' gen. nov., sp. nov., an endosymbiont of arbuscular mycorrhizal fungi. Int J Syst Evol Microbiol 53:121-124. doi: 10.1099/ijs.0.02382-0.

Blin K, Shaw S, Steinke K, Villebro R, Ziemert N, Lee SY, Medema MH, Weber T. 2019. antiSMASH 5.0: updates to the secondary metabolite genome mining pipeline. Nucleic Acids Research 47:W81-W87. doi: 10.1093/nar/gkz310.

Carter ME, Carpenter SCD, Dubrow ZE, Sabol MR, Rinaldi FC, Lastovetsky OA, Mondo SJ, Pawlowska TE, Bogdanove AJ. 2020. A TAL effector-like protein of an endofungal bacterium increases the stress tolerance and alters the transcriptome of the host. Proceedings of the National Academy of Sciences 117:17122. doi: 10.1073/pnas.2003857117.

Chiang YM, Szewczyk E, Davidson AD, Keller N, Oakley BR, Wang CCC. 2009. A gene cluster containing two fungal polyketide synthases encodes the biosynthetic pathway for a polyketide, asperfuranone, in Aspergillus nidulans. J Am Chem Soc 131:2965-2970. doi: 10.1021/ja8088185.

Cianfanelli FR, Monlezun L, Coulthurst SJ. 2016. Aim, load, fire: the type VI secretion system, a bacterial nanoweapon. Trends Microbiol 24:51-62. doi: 10.1016/j.tim.2015.10.005.

Conesa A, Götz S, García-Gómez JM, Terol J, Talón M, Robles M. 2005. Blast2GO: A universal tool for annotation, visualization and analysis in functional genomics research. Bioinformatics 21:3674-3676. doi: 10.1093/bioinformatics/bti610.

Desiro A, Faccio A, Kaech A, Bidartondo MI, Bonfante P. 2015. Endogone, one of the oldest plant-associated fungi, host unique Mollicutes-related endobacteria. New Phytol. 205:1464e1472. doi: 10.1111/nph.13136.

Dobin A, Davis CA, Schlesinger F, Drenkow J, Zaleski C, Jha S, Batut P, Chaisson M, Gingeras TR. 2013. STAR: ultrafast universal RNA-seq aligner. Bioinformatics 29:15-21. doi: 10.1093/bioinformatics/bts635.

Douglas LM, Wang HX, Konopka JB. 2013. The MARVEL domain protein Nce102 regulates actin organization and invasive growth of Candida albicans. mBio 4:e00723-13. doi: 10.1128/mBio.00723-13.

Fu C, Iyer P, Herkal A, Abdullah J, Stout A, Free SJ. 2011. Identification and characterization of genes required for cell-to-cell fusion in Neurospora crassa. Eukaryot Cell 10:1100-1109. doi: 10.1128/EC.05003-11.

Gao M, Glenn AE, Blacutt AA, Gold SE. 2017. Fungal lactamases: their occurrence and function. Frontiers Microbiol 8:1775. doi: 10.3389/fmicb.2017.01775.

Glenn AE, Davis CB, Gao M, Gold SE, Mitchell TR, Proctor RH, Stewart JE, Snook ME. 2016. Two Horizontally Transferred Xenobiotic Resistance Gene Clusters Associated with Detoxification of Benzoxazolinones by Fusarium Species. PloS one 11:e0147486. doi: 10.1371/journal.pone.0147486.

Goldberg MB. 2001. Actin-based motility of intracellular microbial pathogens. Microbiol Mol Biol Rev 65:595-626. doi: 10.1128/MMBR.65.4.595-626.2001. 
Guo H, Glaeser SP, Alabid I, Imani J, Haghighi H, Kämpfer P, Kogel KH. 2017a. The abundance of endofungal bacterium Rhizobium radiobacter (syn. Agrobacterium tumefaciens) increases in its fungal host Piriformospora indica during the tripartite sebacinalean symbiosis with higher plants. Frontiers Microbiol 8:629. doi: 10.3389/fmicb.2017.00629.

Guo M, Tan L, Nie X, Zhang Z. 2017b. A class-II myosin is required for growth, conidiation, cell wall integrity and pathogenicity of Magnaporthe oryzae. Virulence 8:1335-1354. doi: 10.1080/21505594.2017.1323156.

Haas B J, Papanicolaou A, Yassour M, Grabherr M, Blood PD, Bowden J, Couger MB, Eccles D, Li B, Lieber M, MacManes MD, Ott M, Orvis J, Pochet N, Strozzi F, Weeks N, Westerman R, Colin TW, Dewey CN, Henschel R, LeDuc RD, Friedman N, Regen A. 2013. De novo transcript sequence reconstruction from RNA-seq using the Trinity platform for reference generation and analysis. Nat Protocols 8:1494-1512. doi: 10.1038/nprot.2013.084.

Hannon GJ. 2010. FASTX-Toolkit. Available at: http://hannonlab.cshl.edu/fastx toolkit/. Hartley SW, Mullikin JC. 2015. QoRTs: a comprehensive toolset for quality control and data processing for RNA-Seq experiments. BMC Bioinformatics 16:224. doi: 10.1186/s12859015-0670-5.

Hoffman MT, Arnold AE. 2008. Geographic locality and host identity shape fungal endophyte communities in cupressaceous trees. Mycological Research 112:331-344. doi: 10.1016/j.mycres.2007.10.014.

Hoffman MT, Arnold AE. 2010. Diverse bacteria inhabit living hyphae of phylogenetically diverse fungal endophytes. Appl Environ Microbiol 76:4063-4075. doi: 10.1128/AEM.02928-09.

Hoffman MT, Gunatilaka MK, Wijeratne K, Gunatilaka L, Arnold AE. 2013. Endohyphal bacterium enhances production of indole-3-acetic acid by a foliar fungal endophyte. PloS one 8:e73132. doi: 10.1371/journal.pone.0073132.

Huerta-Cepas J, Forslund K, Coelho LP, Szklarcyk D, Jensen LJ, von Mering C, Bork P. 2017. Fast genome-wide functional annotation through orthology assignment by eggNOG-Mapper. Mol Biol Evol 34:2115-2122. doi: 10.1093/molbev/msx148.

Izumi H, Anderson IC, Alexander IJ, Killham K, Moore ER. 2006. Endobacteria in some ectomycorrhiza of Scots pine (Pinus sylvestris). FEMS Microbiol Ecol 56:34-43. doi: 10.1111/j.1574-6941.2005.00048.x.

Jones P, Binns D, Chang HY, Fraser M, Li W, McAnulla C, McWilliam H, Maslen J, Mitchell A, Nuka G, Pesseat S, Quinn AF, Sangrador A, Scheremetjew M, Yong SY, Lopez R, Hunter S. 2014. InterProScan 5: genome-scale protein function classification. Bioinformatics 30:1236-1240. doi: 10.1093/bioinformatics/btu031.

Käll L, Krogh A, Sonnhammer ELL. 2004. A combined transmembrane topology and signal peptide prediction method. J Mol Biol 338:1027-1036. doi: 10.1016/j.jmb.2004.03.016.

Kitagaki H, Wu H, Shimoi H, Ito K. 2002. Two homologous genes, DCW1 (YKL046c) and DFG5, are essential for cell growth and encode glycosylphosphatidylinositol (GPI)-anchored membrane proteins required for cell wall biogenesis in Saccharomyces cerevisiae. Mol Microbiol 46:1011-1022. doi: 10.1046/j.1365-2958.2002.03244.x.

Lackner G, Moebius N, Hertweck C. 2011. Endofungal bacterium controls its host by an hrp type III secretion system. ISME J 5:252-261. doi: 10.1038/ismej.2010.126.

Lastovetsky OA, Gaspar ML, Mondo SJ, LaButti KM, Sandor L, Grigoriev IV, Henry SA, Pawlowska TE. 2016. Lipid metabolic changes in an early divergent fungus govern the 
802

establishment of a mutualistic symbiosis with endobacteria. Proc Natl Acad Sci USA 113:15102-15107. doi: 10.1073/pnas.1615148113.

Lastovetsky OA, Krasnovsky LD, Qin X, Gaspar ML, Gryganskyi AP, Huntemann M, Clum A, Pillay M, Palaniappan K, Varghese N, Mikhailova N, Stamatis D, Reddy TBK, Daum C, Shapiro N, Ivanova N, Kyrpides N, Woyke T, Pawlowska TE. 2020. Molecular dialogues between early divergent fungi and bacteria in an antagonism versus a mutualism. mBio 11:e2088-02020. doi: 10.1128/mBio.02088-20.

Li H, Handsaker B, Wysoker A, Fennell T, Ruan J, Homer N, Marth G, Abecasis G, Durbin R, 100 Genome Project Data Processing Subgroup. 2009. The sequence alignment/map format and SAMtools. Bioinformatics 25:2078-2079. doi: 10.1093/bioinformatics/btp352.

Liao Y, Smyth GK, Shi W. 2014. featureCounts: an efficient general purpose program for assigning sequence reads to genomic features. Bioinformatics 30:923-930. doi: 10.1093/bioinformatics/btt656.

Lumini E, Bianciotto V, Jargeat P, Novero M, Salvioli A, Faccio A, Becard G, Bonfante P. 2007. Presymbiotic growth and sporal morphology are affected in the arbuscular mycorrhizal fungus Gigaspora margarita cured of its endobacteria. Cellular Microbiol 9:1716-1729. doi: 10.1111/j.1462-5822.2007.00907.x.

Lynch JB, Hsiao EY. 2019. Microbiomes as sources of emergent host phenotypes. Science 365:1405-1409. doi: 10.1126/science.aay0240.

MacDonald RM, Chandler MR, Mosse B. 1982. The occurrence of bacterium-like organelles in vesicular-arbuscular mycorrhizal fungi. New Phytol 90:659-663. doi: 10.1111/j.14698137.1982.tb03275.x.

Moebius N, Uzum Z, Dijksterhuis J, Lackner G, Hertweck C. 2014. Active invasion of bacteria into living fungal cells. eLife 3:e03007. doi: 10.7554/eLife.03007.

Mondo SJ, Lastovetsky OA, Gaspar ML, Schwardt NH, Barber CC, Riley R, Sun H, Grigoriev IV, Pawlowska TE. 2017. Bacterial endosymbionts influence host sexuality and reveal reproductive genes of early divergent fungi. Nat Commun 8:1843. doi: 10.1038/s41467-01702052-8.

Obasa K, White FF, Fellers J, Kennelly M, Liu S, Katz B, Tomich J, Moore D, Shinogle H, Kelley K. 2017. A dimorphic and virulence-enhancing endosymbiont bacterium discovered in Rhizoctonia solani. Phytobiomes 1:14-23. doi: 10.1094/PBIOMES-08-16-0005-R.

Osterman IA, Dikhtyar YY, Bogdanov AA, Dontsova OA, Sergiev PV. 2015. Regulation of flagellar gene expression in bacteria. Biochemistry (Moscow) 80:1447-1456. doi: 10.1134/S000629791511005X.

Palmer JM, Stajich J. 2020. Funannotate v1.8.1: Eukaryotic genome annotation. Zenodo. doi: 10.5281/zenodo.4054262.

Partida-Martinez LP, Hertweck C. 2005. Pathogenic fungus harbors endosymbiotic fungus for toxin production. Nature 437:884-888. doi: 10.1038/nature03997.

Partida-Martinez LP, Monajembashi S, Greulich KO, Hertweck C. 2007a. Endosymbiontdependent host reproduction maintains bacterial-fungal mutualism. Curr Biol 17:773-777. doi: 10.1016/j.cub.2007.03.039.

Partida-Martinez LP, Groth I, Schmitt I, Richter W, Roth M, Hertweck C. 2007b. Burkholderia rhizoxinica sp. nov. and Burkholderia endofungorum sp. nov., bacterial endosymbionts of the plant-pathogenic fungus Rhizopus microsporus. Int J Syst Evol Microbiol 57 2583-2590. doi: 10.1099/ijs.0.64660-0 
Pawlowska TE, Gaspar ML, Lastovetsky OA, Mondo SJ, Real-Ramirez I, Shakya E, Bonfante P. 2018. Biology of fungi and their bacterial endosymbionts. Annual Rev Phytopathol 56:289309. doi: 10.1146/annurev-phyto-080417-045914.

Perlin MH, Andrews J, San Toh S. 2014. Essential letters in the fungal alphabet: ABC and MFS transporters and their roles in survival and pathogenicity. Adv Genet 85:201-253. doi: 10.1016/B978-0-12-800271-1.00004-4.

Pinto F, Thapper A, Sontheim W, Lindblad P. 2009. Analysis of current and alternative phenol based RNA extraction methodologies for cyanobacteria. BMC Mol Biol 10:79. doi: 10.1186/1471-2199-10-79.

R Core Team (2020). R: A language and environment for statistical computing. R Foundation for Statistical Computing, Vienna, Austria. URL https://www.R-project.org/.

Rawlings ND, Barrett AJ, Thomas PD, Huang X, Bateman A, Finn RD. 2018. The MEROPS database of proteolytic enzymes, their substrates and inhibitors in 2017 and a comparison with peptidases in the PANTHER database. Nucleic Acids Research 46:D624-D632. doi: 10.1093/nar/gkx1134.

Renshaw H, Vargas-Muñiz JM, Richards AD, Asfaw YG, Juvvadi PR, Steinbach WJ. 2016. Distinct roles of myosins in Aspergillus fumigatus hyphal growth and pathogenesis. Infection and Immunity 84:1556-1564. doi: 10.1128/IAI.01190-15.

Reza MH, Shah H, Manjrekar J, Chattoo BB. 2016. Magnesium uptake by CorA transporters is essential for growth, development and infection in the rice blast fungus Magnaporthe oryzae. PloS one 11:e0159244-e0159244. doi: 10.1371/journal.pone.0159244.

Saint-Macary ME, Barbisan C, Gagey MJ, Frelin O, Beffa R, Lebrun MH, Droux M. 2014. Methionine biosynthesis is essential for infection in the rice blast fungus Magnaporthe oryzae. PloS one 10:e0111108. doi:10.1371/journal. pone.0111108.

Savary S, Willocquet L, Pethybridge SJ, Esker P, McRoberts N, Nelson A. 2019. The global burden of pathogens and pests on major food crops. Nat Ecol Evol 3:430-439. doi: 10.1038/s41559-018-0793-y.

Seppey M, Manni M, Zdobnov EM. 2019. BUSCO: Assessing Genome Assembly and Annotation Completeness, p 227-245. In Kollmar M (ed), Gene Prediction: Methods and Protocols. Springer, New York, NY.

Shaffer JP, Sarmiento C, Zalamea PC, Gallery RE, Davis AS, Baltrus DA, Arnold AE. 2016. Diversity, specificity, and phylogenetic relationships of endohyphal bacteria in Fungi that inhabit tropical seeds and leaves. Frontiers Ecol Evol 4:116. doi: 10.3389/fevo.2016.00116.

Shaffer JP, U'Ren JM, Gallery RE, Baltrus DA, Arnold AE. 2017. An endohyphal bacterium (Chitinophaga, Bacteroidetes) alters carbon source use by Fusarium keratoplasticum (F. solani Species Complex, Nectriaceae). Frontiers Microbiol 8:350. doi: 10.3389/fmicb.2017.00350.

Shaffer JP, Zalamea PC, Sarmiento C, Gallery RE, Dalling JW, Davis AS, Baltrus DA, Arnold AE. 2018. Context-dependent and variable effects of endohyphal bacteria on interactions between fungi and seeds. Fungal Ecol 36:117-127. doi: 10.1016/j.funeco.2018.08.008.

Shyntum DY, Venter SN, Moleleki LC, Toth I, Coutinho TA. 2014. Comparative genomics of type VI secretion systems in strains of Pantoea amanitis from different environments. BMC Genomics 15:163. doi: 10.1186/1471-2164-15-163.

Si M, Zhao C, Burkinshaw B, Zhang B, Wei D, Wang Y, Dong TG, Shen X. 2016. Manganese scavenging and oxidative stress response mediated by type VI secretion system in 

10.1073/pnas.1614902114.

Solomon PS, Nielsen PS, Clark AJ, Oliver RP. 2000. Methionine synthase, a gene required for methionine synthesis, is expressed in planta by Cladosporium fulvum. Mol Plant Pathol 1:315-323. doi: 10.1046/j.1364-3703.2000.00035.x.

Spraker JE, Sanchez LM, Lowe TM, Dorrestein PC, Keller NP. 2016. Ralstonia solanacearum lipopeptide induces chlamydospore development in fungi and facilitates bacterial entry into fungal tissues. ISME J 10:2317-2330. doi: 10.1038/ismej.2016.32.

Steffan BN, Venkatesh N, Keller NP. 2020. Let's get physical: bacterial-fungal interactions and their consequences in agriculture and health. J Fungi 6:243. doi: 10.3390/jof6040243.

Walvekar AS, Laxman S. 2019. Methionine at the heart of anabolism and signaling: perspective from budding yeast. Frontiers Microbiol 10:2624. doi: 10.3389/fmicb.2019.02624.

Yin Y, Mao X, Yang J, Chen X, Mao F, Xu Y. 2012. dbCAN: a web resource for automated carbohydrate-active enzyme annotation. Nucleic Acids Research 40:W445-W451. doi: 10.1093/nar/gks479.

Zhang X, Han L, Wang Q, Zhang C, Yu Y, Tian J, Kong Z. 2019. The host actin cytoskeleton channels rhizobia release and facilitates symbiosome accommodation during nodulation in Medicago truncatula. New Phytol 221:1049-1059. doi: 10.1111/nph.15423.

Zimin AV, Marçais G, Puiu D, Roberts M, Salzburg SL, Yorke JA. 2013. The MaSuRCA genome assembler. Bioinformatics 29:2669-2677. doi: 10.1093/bioinformatics/btt476.

Zimin AV, Puiu D, Luo MC, Zhu T, Koren S, Marçais G, Yorke JA, Dvorák J, Salzberg SL. 2017. Hybrid assembly of the large and highly repetitive genome of Aegilopsis tauschii, a progenitor of bread wheat, with the MaSuRCA mega-reads algorithm. Genome Research

916 27:787-792. doi: 10.1101/gr.213405.116. 


\section{TABLES AND FIGURES}

918 TABLE 1. Final statistics for the genome of Pestalotiopsis sp. 9143.

Genome assembly:

\begin{tabular}{|r|l|}
\hline Genome assembly: & \\
\hline Unique scaffolds & 104 \\
\hline Final assembly size & $46,255,514 \mathrm{bp}$ \\
Masked repeats & $1,379,150 \mathrm{bp}(2.98 \%)$ \\
\hline N50 & 817,980 \\
\hline GC content & $52 \%$ \\
\hline Gene models: & \\
\hline Protein-coding & 13,076 chromosomal; 40 mitochondrial \\
\hline tRNAs & 201 chromosomal; 65 mitochondrial \\
\hline
\end{tabular}

919

920 
921 TABLE 2. Differentially expressed genes of Pestalotiopsis sp. 9143 annotated with the

922 enriched GO terms associated with the cytoskeleton and cell wall and their top hit using PSI-

923 BLAST.

\begin{tabular}{|c|c|c|c|c|c|}
\hline Gene ID & Annotation & L2FC & FDR & PSI-BLAST & Organism \\
\hline KJ359_001200 & hypothetical protein & 4.42 & $1.83 \mathrm{E}-05$ & Endochitinase & Manduca sexta \\
\hline KJ359_008820 & hydrolase 76 protein & 2.94 & $2.54 \mathrm{E}-04$ & $\begin{array}{l}\text { Mannan endo-1,6- } \\
\text { alpha-mannosidase }\end{array}$ & $\begin{array}{l}\text { Saccharomyces } \\
\text { cerevisiae }\end{array}$ \\
\hline KJ359_008681 & hypothetical protein & 2.68 & $1.73 \mathrm{E}-04$ & Kinesin & $\begin{array}{l}\text { Cylindrotheca } \\
\text { fusiformis }\end{array}$ \\
\hline KJ359_002383 & hypothetical protein & 2.62 & $9.43 \mathrm{E}-05$ & Chitotriosidase-1 & Homo sapiens \\
\hline KJ359_012237 & hydrolase 76 protein & 1.67 & $7.40 \mathrm{E}-04$ & $\begin{array}{l}\text { Mannan endo-1,6- } \\
\text { alpha-mannosidase }\end{array}$ & $\begin{array}{l}\text { Saccharomyces } \\
\text { cerevisiae }\end{array}$ \\
\hline KJ359_000369 & $\begin{array}{l}\text { Tubulin beta chain } \\
\text { (Beta tubulin) }\end{array}$ & 1.38 & 4.20E-04 & Tubulin beta chain & $\begin{array}{l}\text { Pestalotiopsis } \\
\text { microspora }\end{array}$ \\
\hline KJ359_011436 & hypothetical protein & 1.32 & 8.61E-03 & Flocculation protein & $\begin{array}{l}\text { Saccharomyces } \\
\text { cerevisiae }\end{array}$ \\
\hline KJ359_012899 & $\begin{array}{l}\text { Chitin synthase, } \\
\text { class } 2\end{array}$ & 1.30 & $1.78 \mathrm{E}-03$ & Chitin synthase 1 & $\begin{array}{l}\text { Neurospora } \\
\text { crassa }\end{array}$ \\
\hline KJ359_009573 & hypothetical protein & 1.28 & $8.75 \mathrm{E}-05$ & Chitin Synthase 6 & Ustilago maydis \\
\hline KJ359_000339 & hypothetical protein & 1.17 & 2.32E-03 & Proline-rich protein & $\begin{array}{l}\text { Saccharomyces } \\
\text { cerevisiae }\end{array}$ \\
\hline KJ359_003752 & $\begin{array}{l}\text { Chitin synthase, } \\
\text { class } 1\end{array}$ & 1.16 & $5.09 \mathrm{E}-03$ & Chitin synthase 3 & $\begin{array}{l}\text { Neurospora } \\
\text { crassa }\end{array}$ \\
\hline KJ359_011288 & alpha-tubulin & 1.12 & $1.65 \mathrm{E}-03$ & $\begin{array}{l}\text { Tubulin alpha-B } \\
\text { chain }\end{array}$ & $\begin{array}{l}\text { Neurospora } \\
\text { crassa }\end{array}$ \\
\hline KJ359_012862 & class II myosin & 1.11 & $8.65 \mathrm{E}-04$ & Myosin 1 & $\begin{array}{l}\text { Magnaporthe } \\
\text { oryzae }\end{array}$ \\
\hline KJ359_010970 & $\begin{array}{l}\text { Myosin type- } 2 \\
\text { heavy chain } 1\end{array}$ & 1.11 & 6.37E-03 & Myosin 2 & $\begin{array}{l}\text { Lachancea } \\
\text { kluyveri }\end{array}$ \\
\hline KJ359_010970 & $\begin{array}{l}\text { Myosin type- } 2 \\
\text { heavy chain } 1\end{array}$ & 1.11 & $6.37 \mathrm{E}-03$ & Myosin 2 & $\begin{array}{l}\text { Saccharomyces } \\
\text { cerevisiae }\end{array}$ \\
\hline KJ359_005696 & hypothetical protein & 1.06 & $1.11 \mathrm{E}-03$ & $\begin{array}{l}\text { Actin-binding } \\
\text { protein }\end{array}$ & $\begin{array}{l}\text { Saccharomyces } \\
\text { exiguus }\end{array}$ \\
\hline KJ359_012986 & hypothetical protein & -3.06 & $1.93 \mathrm{E}-13$ & $\begin{array}{l}\text { Mannan endo-1,6- } \\
\text { alpha-mannosidase }\end{array}$ & $\begin{array}{l}\text { Saccharomyces } \\
\text { cerevisiae }\end{array}$ \\
\hline
\end{tabular}

924

925 

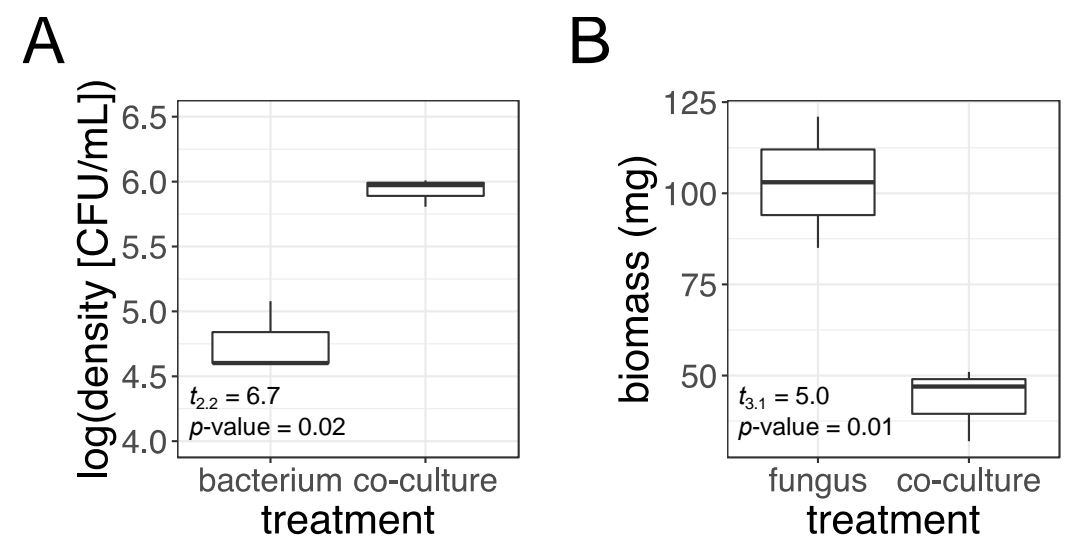

927 FIG 1 Differences in microbial density/biomass during co-culture vs. axenic growth. (A) Density

928 (colony forming units $[\mathrm{CFU}] / \mathrm{mL}$ ) of free-living Luteibacter sp. 9143 (i.e., cells not associated

929 with fungal mycelium) when grown axenically (bacterium) vs. with Pestalotiopsis sp. 9143 (co-

930 culture). (B) Biomass (mg) of Pestalotiopsis sp. 9143 when grown axenically (fungus) vs. with

931 Luteibacter sp. 9143 (co-culture). The same volume of liquid growth media was initially added

932 to each sample. Biomass represents dry mass obtained by filtering cultures to remove the growth

933 medium and lyophilizing the fresh biomass that was retained.

934 
A

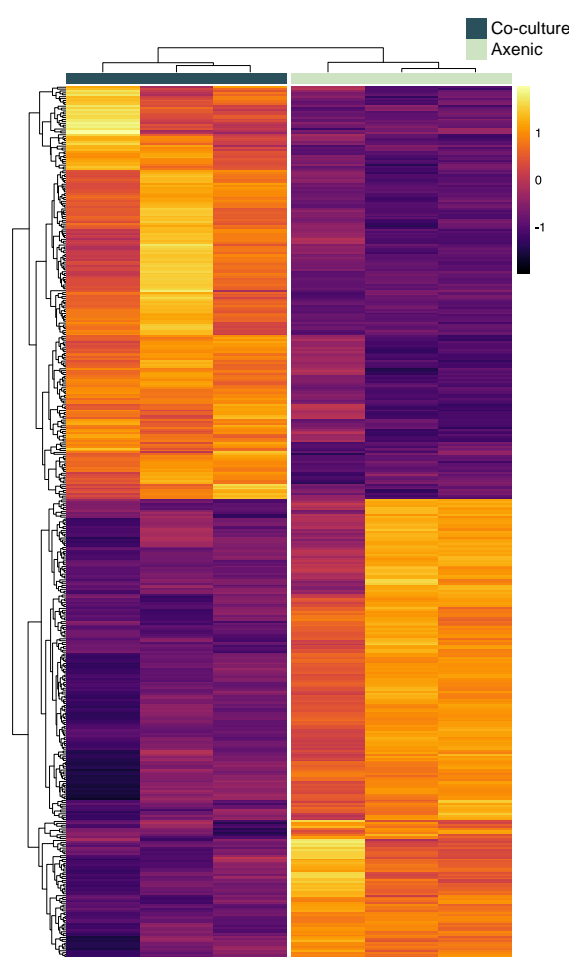

B

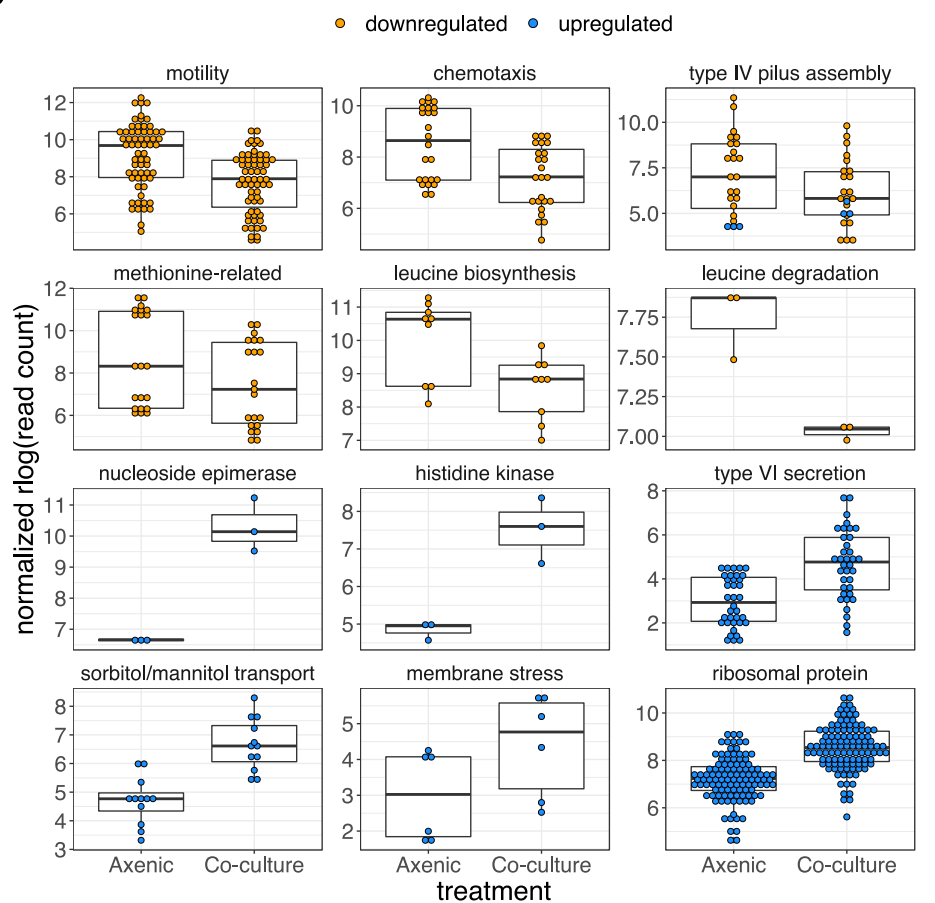

936 FIG 2 Summary of differentially expressed genes in Luteibacter sp. 9143 in co-culture with

937 Pestalotiopsis sp. 9143 compared to axenic growth. (A) Heatmap showing the log-fold-change

938 (i.e., deseq2 rlog-normalized) for each gene (rows) for each biological replicate (columns). (B)

939 Normalized read counts for axenic vs. co-cultured Luteibacter sp. 9143 for specific groups of

940 genes. Methionine-related genes include those for methionine biosynthesis, acquisition, and

941 conversion. Each point represents the read count for a given gene from one replicate culture of a

942 given treatment of Luteibacter sp. 9143 (i.e., axenic vs. co-culture), thus three points are present

943 for each gene. 


\section{A}
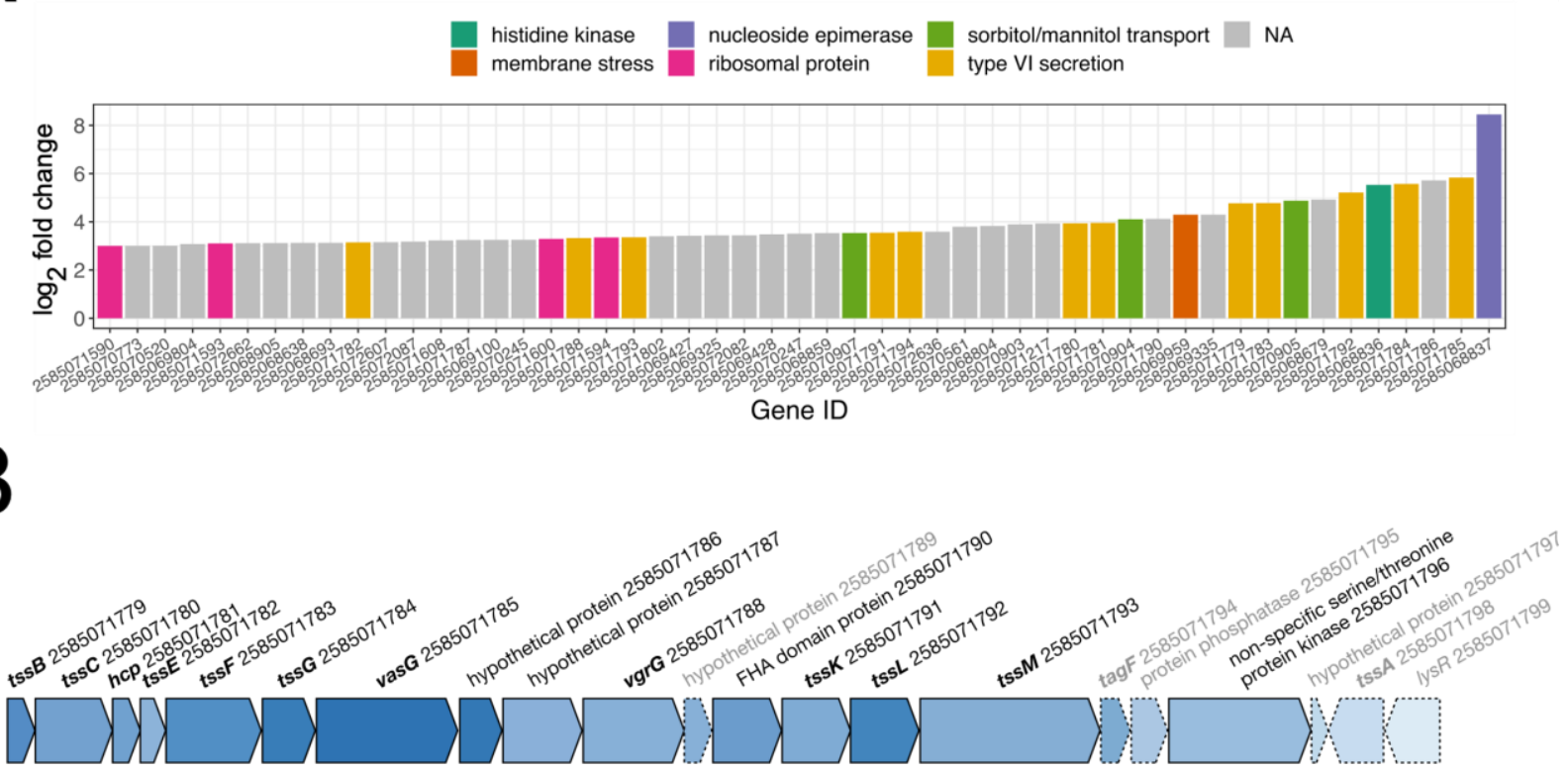

946 FIG 3 Upregulation of type VI secretion system-related genes in Luteibacter sp. 9143 when in

947 co-culture with Pestalotiopsis sp. 9143. (A) $\log _{2}$ fold change (L2FC) for significantly highly

948 upregulated genes (i.e., adjusted $p$-value $\leq 0.01, \mathrm{~L} 2 \mathrm{FC} \geq 3.0$ ), highlighting important groups of

949 genes including those associated with the type VI secretion system (T6SS). (B) Physical map

950 showing proximity of genes associated with the T6SS in bold font. Colors indicate L2FC in co-

951 cultured vs. axenic Luteibacter sp. 9143. Genes annotated in gray and with hashed lines were

952 neutrally regulated and all others were upregulated. 

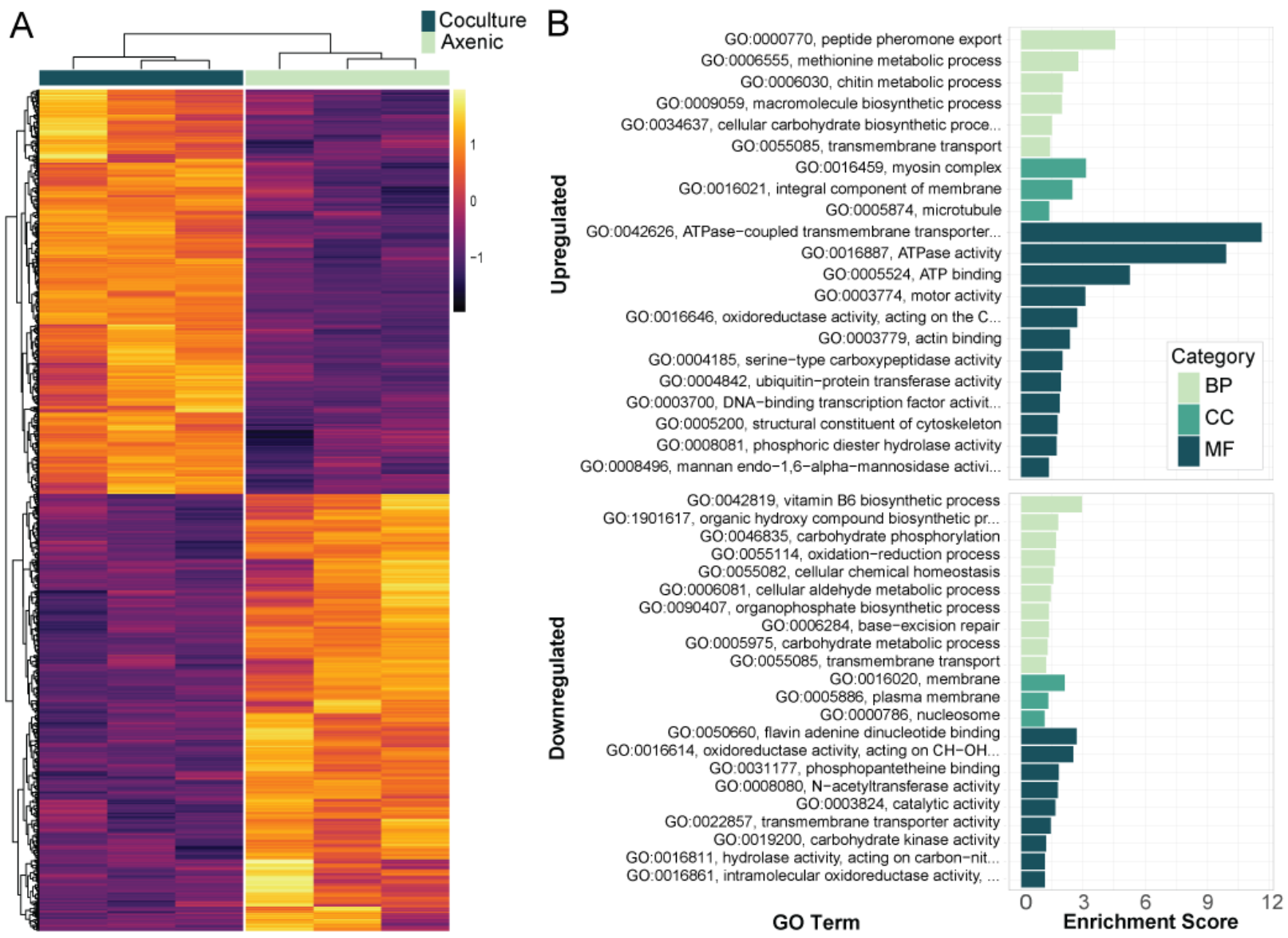

955 FIG 4 Differentially expressed genes and enriched GO terms in Pestalotiopsis sp. 9143 when in

956 co-culture with Luteibacter sp. 9143. (A) Heatmap of regularized log (rlog) transformed counts

957 showing the differentially expressed genes identified with DESeq2 for a false discovery rate of

958 0.01. Three biological replicates are shown for co-cultured and axenic fungal growth. (B)

959 Enriched Gene Ontology (GO) terms among the up- or downregulated differentially expressed

960 genes in co-cultured fungus; biological processes (BP), cellular component (CC), and molecular

961 function (MF). Enrichment analysis was done using the topGO R package's weight01 algorithm

962 and Fisher's exact test for a $p$-value $<0.05$. 
A

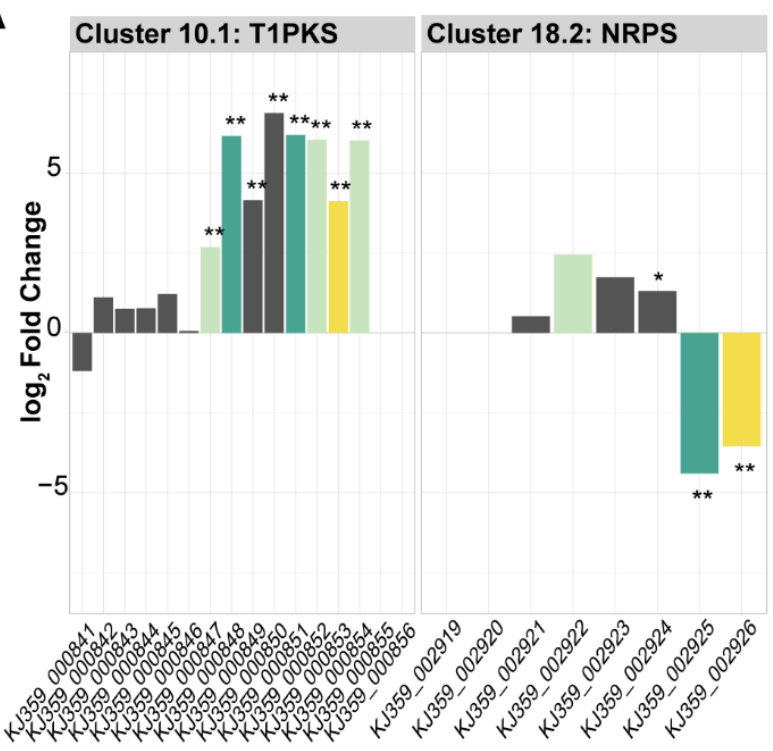

Gene Identifier

\section{Predicted Gene Role \\ core additional transport \#other \\ biosynthetic biosynthetic}

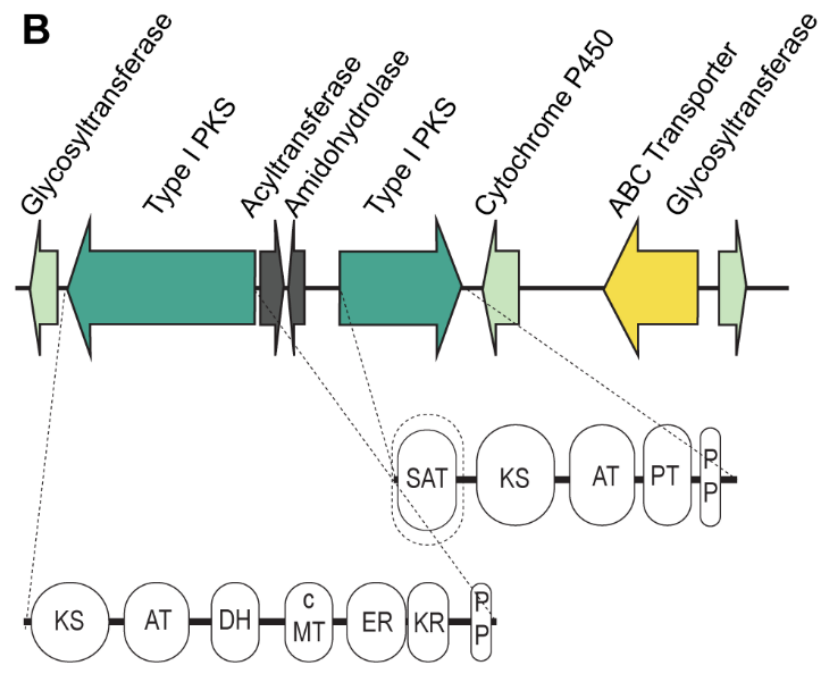

C

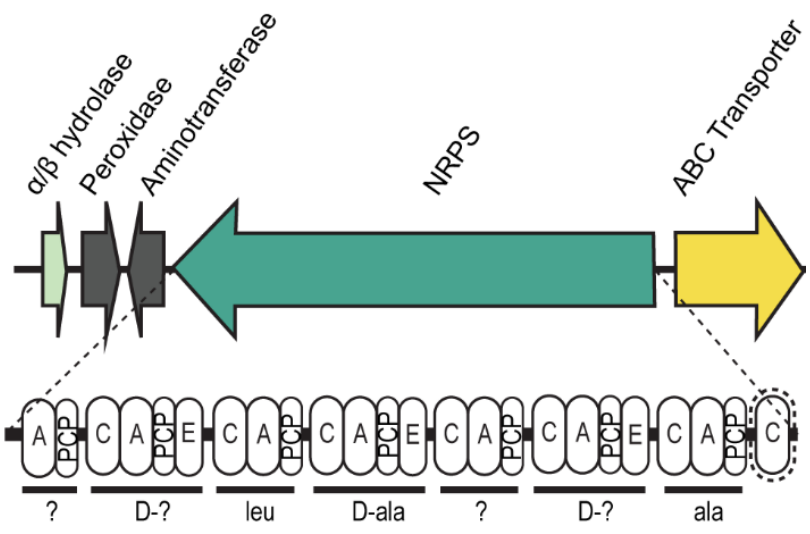


965 FIG 5 Differential expression of genes within two secondary metabolite clusters of

966 Pestalotiopsis sp. 9143. (A) $\log _{2}$ fold change of the genes within Cluster 10.1 and Cluster 18.2

967 color coded by predicted gene role. Two asterisks $(* *)$ designate differentially expressed (DE)

968 genes with FDR<0.01; one asterisk (*) designates DE genes with FDR<0.05. (B) Diagram of

969 part of the Cluster 10.1 Type I polyketide synthase (PKS) locus with putative gene products,

970 including PKS domains: ketosynthase (KS), acyltransferase (AT), phosphopanetheine (PP), acyl

971 carrier protein (ACP) dehydratase (DH), enoylreductase (ER), ketoreductase (KR), carbon

972 methyltransferase (cMT), product template (PT), and a N-terminal ACP transacylase similar to

973 the one involved in aflatoxin biosynthesis (SAT). (C) Diagram of part of the Cluster 18.2

974 nonribosomal peptide synthase (NRPS) locus with putative gene products, including NRPS

975 domains: condensation (C), AMP-binding (A), epimerization (E), and peptidyl-carrier protein

976 (PCP). Bars underneath groups of ovals indicate NRPS modules and the amino acids they are

977 predicted to load: alanine (ala) and leucine (leu). Incomplete modules or predictions are indicated

978 with a question mark (?). 